\title{
EFFICIENT PLACEMENT SCHEMES TO FULLY UTILIZE PEER UPSTREAM BANDWIDTH
}

\begin{abstract}
A THESIS SUBMITTED TO THE GRADUATE DIVISION OF THE UNIVERSITY OF HAWAI'I IN PARTIAL FULFILLMENT OF THE REQUIREMENTS FOR THE DEGREE OF
\end{abstract}

MASTER OF SCIENCE

IN

ELECTRICAL ENGINEERING

DECEMBER 2006

By

Hui min Zeng

Thesis Committee:

Yingfei Dong, Chairperson

Tep Dobry

Galen Sasaki 
We certify that we have read this thesis and that, in our opinion, it is satisfactory in scope and quality as a thesis for the degree of Master of Science in Electrical

\section{Engineering.}

\section{THESIS COMMITTEE}
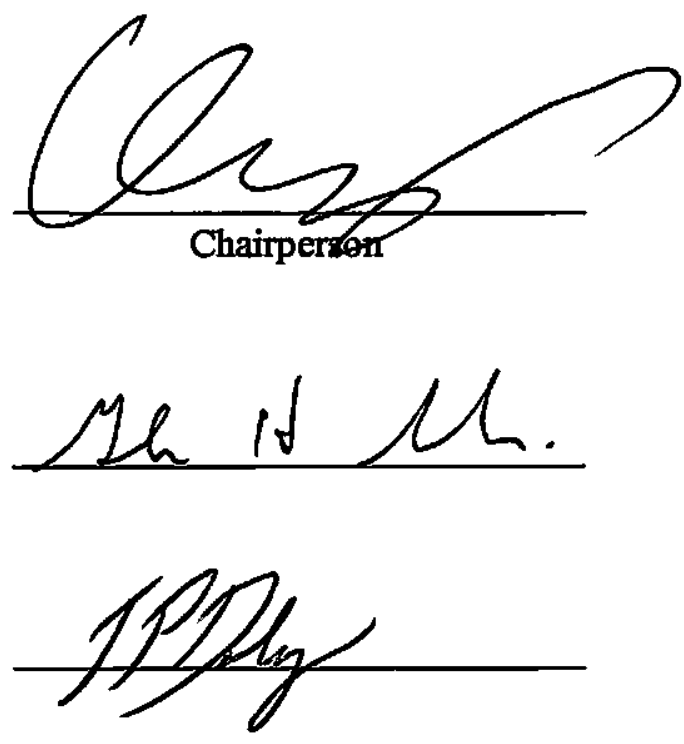


\section{ACKNOWLEDGMENTS}

I am grateful to my advisor Dr. Yingfei Dong for the invaluable guidance and tremendous patience throughout my thesis research, to my committee members, Dr. Tep Dobry and Dr. Galen Sasaki for the support and advice, and to my parents and for their love and blessings. 


\begin{abstract}
Efficiently utilizing limited peer upstream bandwidth is one of the key challenges in P2P streaming. Many mechanisms have been proposed to address this issue by considering peer availability, data redundancy, and priority encoding schemes. In this thesis, we investigate this issue from a unique aspect: how to place video data at peers with various upstream capacities in order to better utilize their upstream bandwidth. We propose several effective placement solutions. Our analysis and simulation results show the efficacy of the proposed solutions.
\end{abstract}




\section{TABLE OF CONTENTS}

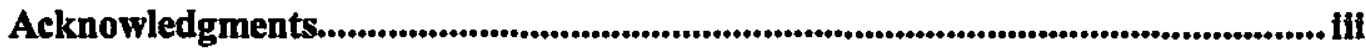

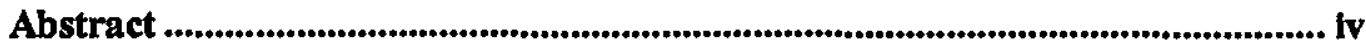

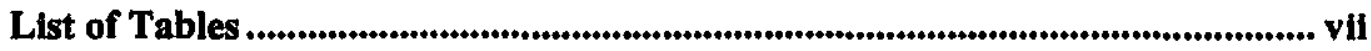

List of Figures............................................................................................................. vili

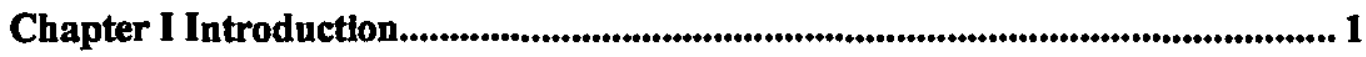

Chapter II System Overview................................................................................. 10

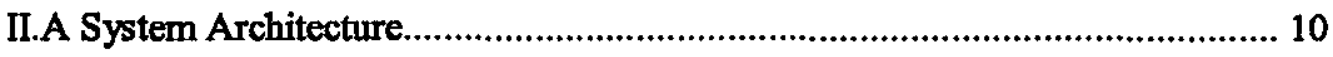

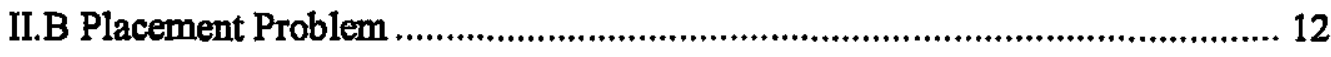

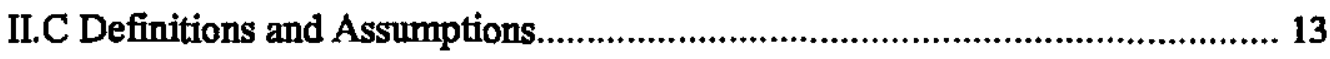

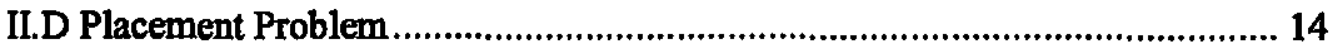

Chapter III Efficient Placement Schemes ................................................................. 16

III.A Two-step Placement Schemes............................................................. 16

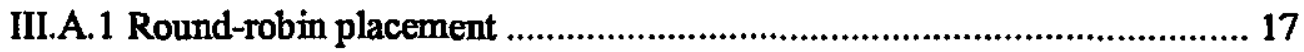

III.A.2 Notations for bandwidth-based placements ..................................... 18

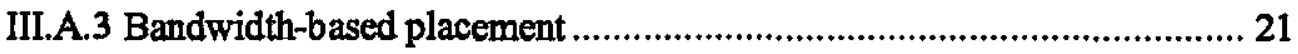

III.B Utilization-based placement............................................................... 23

III.C Placement Adjustment Scheme ............................................................. 24

III.D Placement Scheme Considering Reliability of Supplying Peers.................. 26

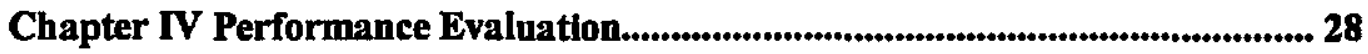

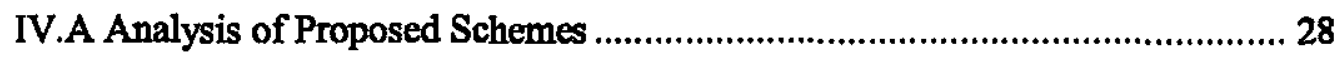

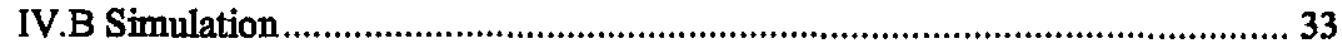

IV.B.1 Evaluation of the Proposed Placement Schemes .................................. 34

IV.B.2 Evaluation of the Placement Adjustment Scheme ................................ 40

IV.B.3 Effect of System Load ..................................................................... 41

IV.B.4 Consideration of the Peer Reliability ................................................. 45 


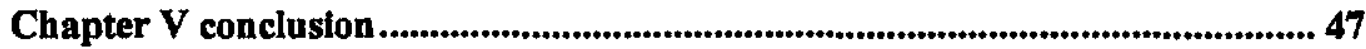

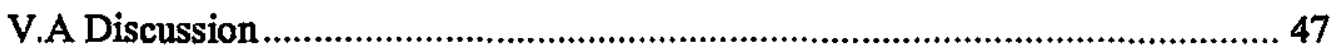

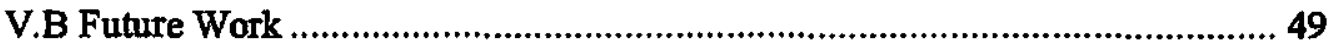

Appendix A: Definitions and/or denotations.................................................51

Appendix B: Pseudocode for the Proposed Placement Schemes ......................... 52

Appendix C: Experiment Results ....................................................................5 58

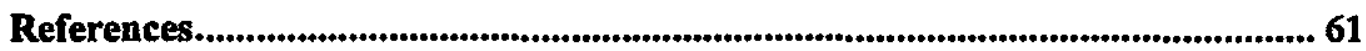




\section{LIST OF TABLES}

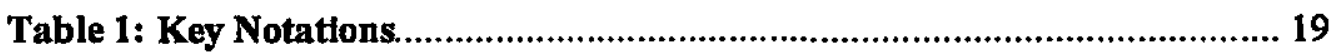

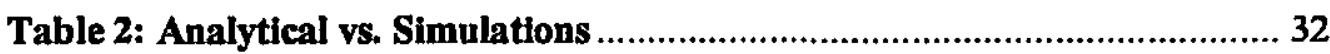

Table 3: Pseudocode for the simulation to measure the utllization of the

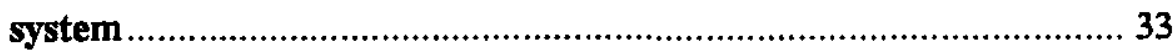

Table 4: Simulation setting for comparing different placements................... 35

Table 5: Difference between utllization-based and round-robin in percentages

Table 6: Difference between bandwidth-based and round-robin in

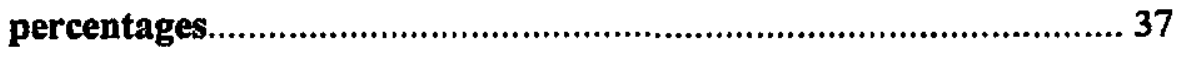

Table 7: Setting for Varying Load Simulation............................................. 42

Table 8: Max \& Min Difierence of the Utilization to Round-robin in

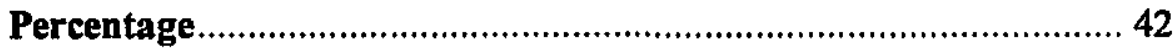




\section{LIST OF FIGURES}

Figure 1: Multiple Description coding and path diversity for video delivery in a

client-server model 3

Figure 2: Video streaming network examples. 6

Figure 3: Hybrid Streaming Architecture .................................................... 11

Figure 4: System Architecture in a Local Network ......................................... 11

Figure 5: Examples of competition with different placements........................... 13

Figure 6: Placement mapping: number of copies and where to put them.............. 15

Figure 7: An example of a collaborative network ....................................... 28

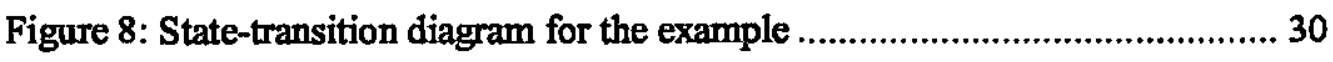

Figure 9: Analytical comparison of the utilization of three placements ................. 32

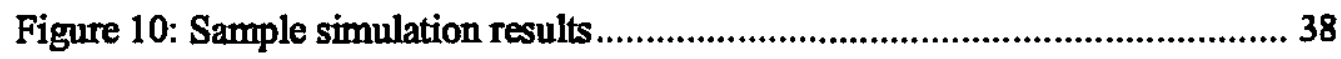

Figure 11: Difference of utilization-based/bandwidth-based and round-robin in

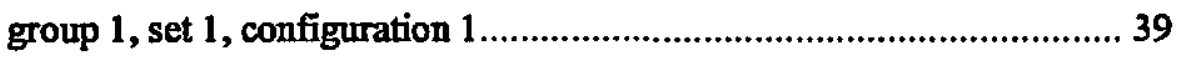

Figure 12: Difference of utilization-based with and without adjustment in utilization Error! Bookmark not defined.

Figure 13: Comparison of utilization-based placement w/ adjustment and other placements w/o adjustment

Figure 14: Comparison of utilization to round-robin scheme, set 1 ................... 43

Figure 15: Rejection rate of the three placements for set $3 \ldots \ldots \ldots \ldots \ldots \ldots \ldots \ldots \ldots \ldots \ldots . . . . . . . . . . . . . . .44$

Figure 16: Comparison of utilization-based placements with set 1,2 \& 3 ........... 44 
Figure 17: Comparison of modified utilization-based and non-modified.............. 45

Figure 18: Difference of the utilization of utilization-based and round-robin

placements in percentages........................................................... 46 


\section{CHAPTER I}

\section{INTRODUCTION}

Video has been an important media for communications and entertainment for many decades. The proliferation of high-speed, broadband networking technologies has made real-time video streaming a reality. In this thesis, we consider an on-demand service of stored videos over the Internet. We investigate a particular design component and propose server schemes to improve the video delivery performance of this component.

Video data is a sequence of video frames. Since neighboring video frames are typically very similar, high compression can be achieved by exploiting the similarity between frames. For example, MPEG is one of the standards to compress a video. It consists of three basic types of coded frames: (i) intra-coded frames, or I-frames, (ii) predicatively coded, or P-frames and (iii) bi-directionally predicted frames, or B-frames. I-frames are coded independently of all other frames. The P-frame is coded based a previously coded frame. The B-frame is coded using both previous and future coded frames.

The basic idea of video streaming is to split the video data into parts, transmit these parts in succession, and enable the user to decode and playback the video as these parts are received, without having to wait for the entire video to be delivered. Video streaming enables simultaneous delivery and playback of the video. This is in contrast to file download where the entire video must be delivered before playback can begin. 
Video streaming over the Internet is difficult because the Internet only offers best effort service. The Internet provides no guarantees on bandwidth or loss rate. The users have various downstream capacities. In addition, the compressed video data is highly vulnerable to errors. A number of coding approaches have been proposed to address these challenges. For example, a scheme known as layered multicast provides a way to cope with the heterogeneous bandwidth of the users. A layered coding scheme produces multiple layers of data, with a base layer that offers low but usable quality, and each additional layer provides further enhancement to the quality. The user can then individually decide how many layers to receive[20]. Another example is Multiple Description Coding (MDC). MD Coding encodes a video data into two or more separate descriptions. MD coding possesses two important properties: (i) each description can be independently decoded with a certain fidelity of the original data, and (2) the multiple descriptions contain complementary information so that the quality of the decoded data improves with the number of descriptions that are received. Since each description can travel the network over a different path as opposed to the default scenarios where the video stream proceed along a single path, it is beneficial to increase the probability that at least one description is received at any point in time[25]. Furthermore, by separating a video data into multiple descriptions, MD coding allows multiple servers to collaboratively deliver a video data to a user, which lowers the requirement for the upstream bandwidth of the servers.

Figure 1 shows an example of a delivery of video on-demand in a client-server 
model, where each video is encoded to multiple descriptions. The server stores a copy of the original video data in MPEG format. When the user requests the video, the server encodes the video data into two descriptions (in this example), and streams the two descriptions to the user via different paths. If the user successfully receives both descriptions, after decoding the descriptions, it can display the video with full quality. If the user receives one of the descriptions, it still can decode that description and playback the video with lower quality.

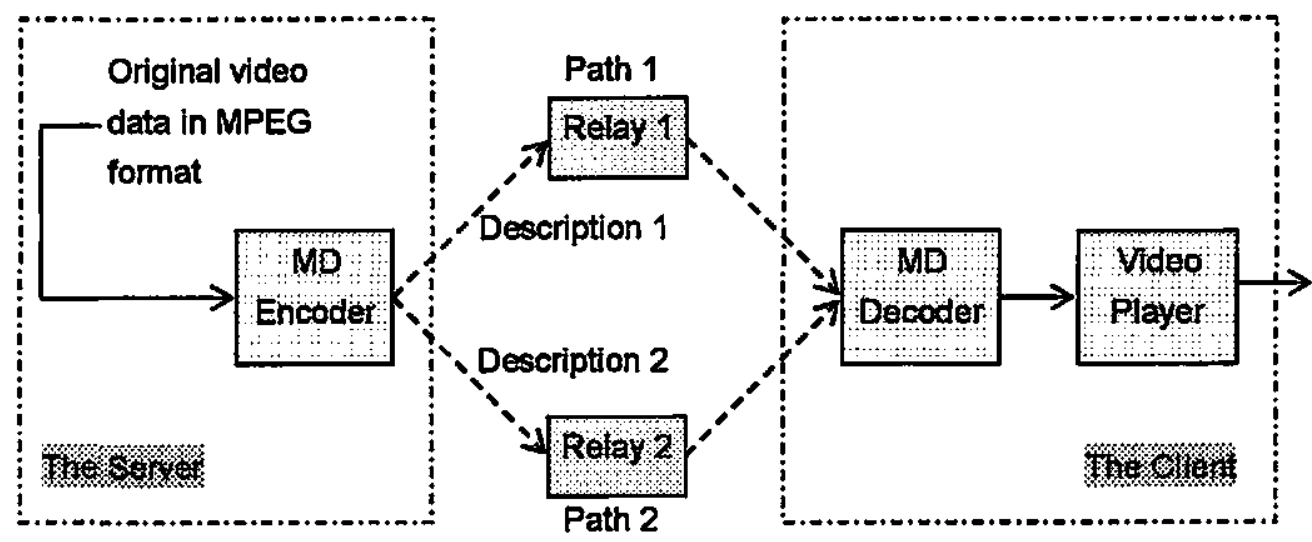

Figure 1: Multiple Description coding and path diversity for video delivery in a

$$
\text { client-server model }
$$

Currently, the networks that support video streaming can be classified into three types: (i) client-server model over unicast or multicast networks, which was mentioned earlier, (ii) Content Distribution Networks (CDN) and (iii) Peer-to-peer streaming networks (P2P).

The client-server model over unicast or multicast networks is one-to-one or one-to-many delivery on the IP layer. IP layer unicast or multicast restrains the delivery in the local area network (LAN). It is simple to manage but not efficient nor 
scalable. For instance, given the capacity $C s$ of the server and the video service rate is numerically set to one, this server can serve no more than $C s$ video requests simultaneously. Figure 2 (a) shows an example of video streaming in client-server model over a unicast network.

CDN is an extension of client-server model - it aims to improve the scalability. CDN is a system of servers networked together across the Internet to cooperate transparently for delivering content to end users. Figure 2 (b) shows an example of video streaming over CDN. CDN dominates the management and distribution of streamed content in the Internet currently. A CDN server has the dedicated storage space and upstream bandwidth for high-quality video streaming. In some applications, more (proxy) servers are sitting at the edge of the network to help the CDN servers to deliver videos. The proxy servers usually cache partial video data and have their own upstream capacity. Multiple Description Streaming Media CDN (MD-CDN) [26] is also proposed to achieve path diversity to improve the performance of the delivery in a lossy environment. However, the server has limited processing power and upstream bandwidth that can support the distribution. CDN servers require a higher investment in broad delivery infrastructures and demand a rather large amount of resources to achieve scalability.

To further improve the scalability and reduce the upstream bandwidth and cache requirements of $\mathrm{CDN}$ and proxy servers, collaborative peers can be another resource that we can utilize. P2P technology has exposed a great potential to provide a scalable and economical solution to content distribution. Many file-downloading have been 
developed and deployed widely, such as Napster [12][13], Gnntella (Bearshare) [12][13][14], FastTrack (Kazaa) [13][15], eDonkey [16], and Bit Torrent [17]. Video streaming over P2P network is a new and active area of research. The basic idea of the video streaming over P2P network is that when a user receives the video, it can start acting as a service peer. Typically, a service peer buffers a partial video data (e.g. one or more descriptions if the data is encoded by MD coding) and several service peers collaboratively serve a request. Figure 2 (c) shows an example of P2P video streaming. However, due to the nature of peers, video streaming over P2P presents a new set of design challenges. First, unlike the CDN servers, the service peers randomly join or leave the network, so that the quality assurance is a problem. Second, the service peers have limited and various upstream bandwidth. A broadband user has an upstream bandwidth up to $256 \mathrm{kbps}$ and the upstream bandwidth for the DSL ranges from $96 \mathrm{kbps}$ to $640 \mathrm{kbps}$ [27]. Many users are DSL users and most of them have a low range of upstream bandwidth.

A near DVD quality video streaming requires a stream rate at least 500kbps [11]. One peer alone is not enough to serve the video request due to insufficient upstream bandwidth. If we encode the video data into eight descriptions, then each description only requires around 64kps. In this case, most of the users are able to serve as service peers to stream one or two descriptions. Now, a new challenge arises - how to fully utilize the upstream bandwidth of the peers? This is one of the main concerns in this thesis. 


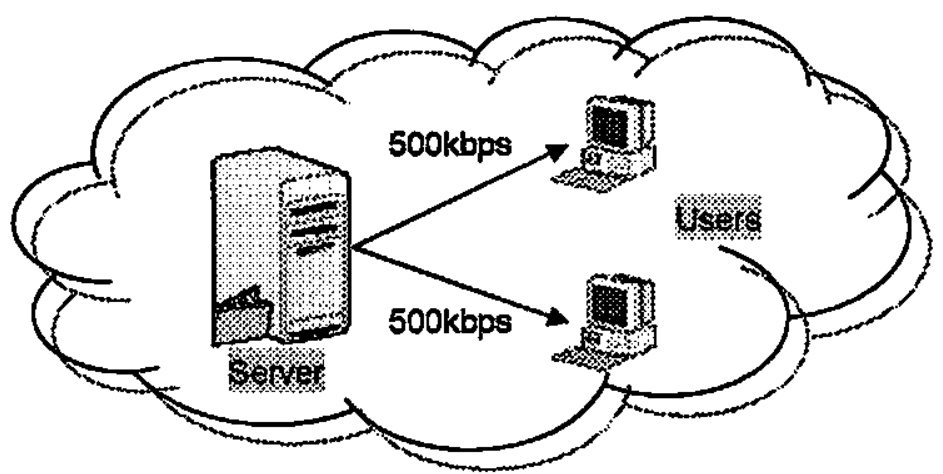

(a) Client-server model

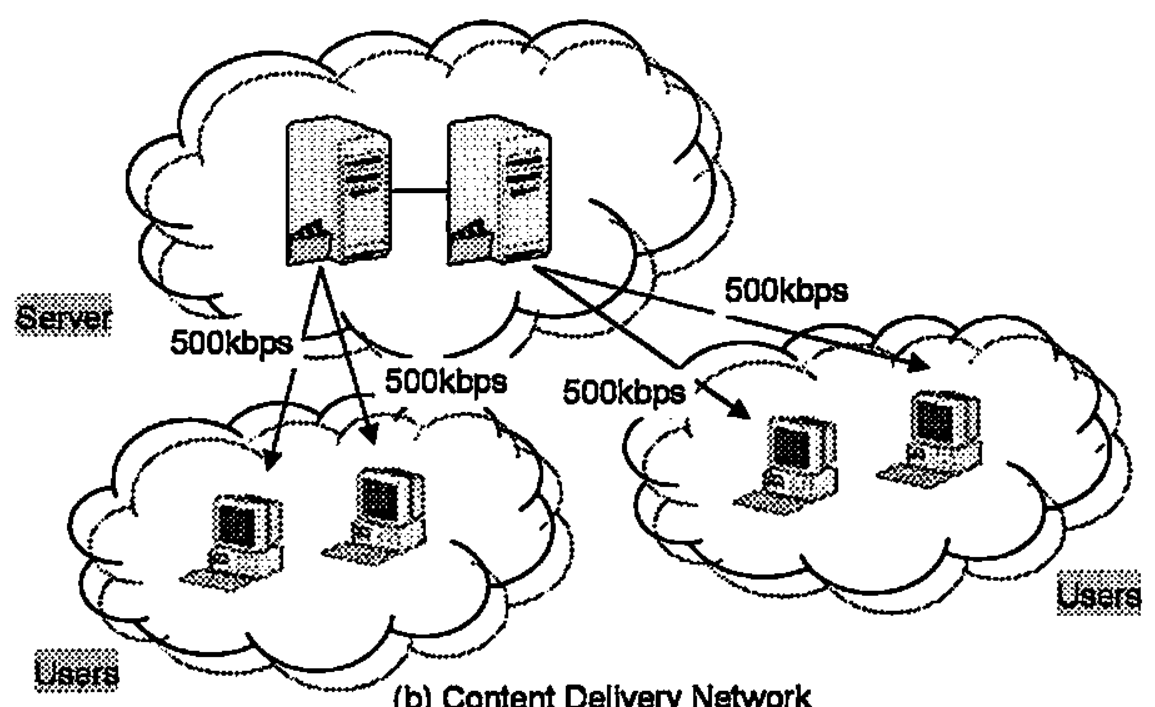

(b) Content Delivery Network

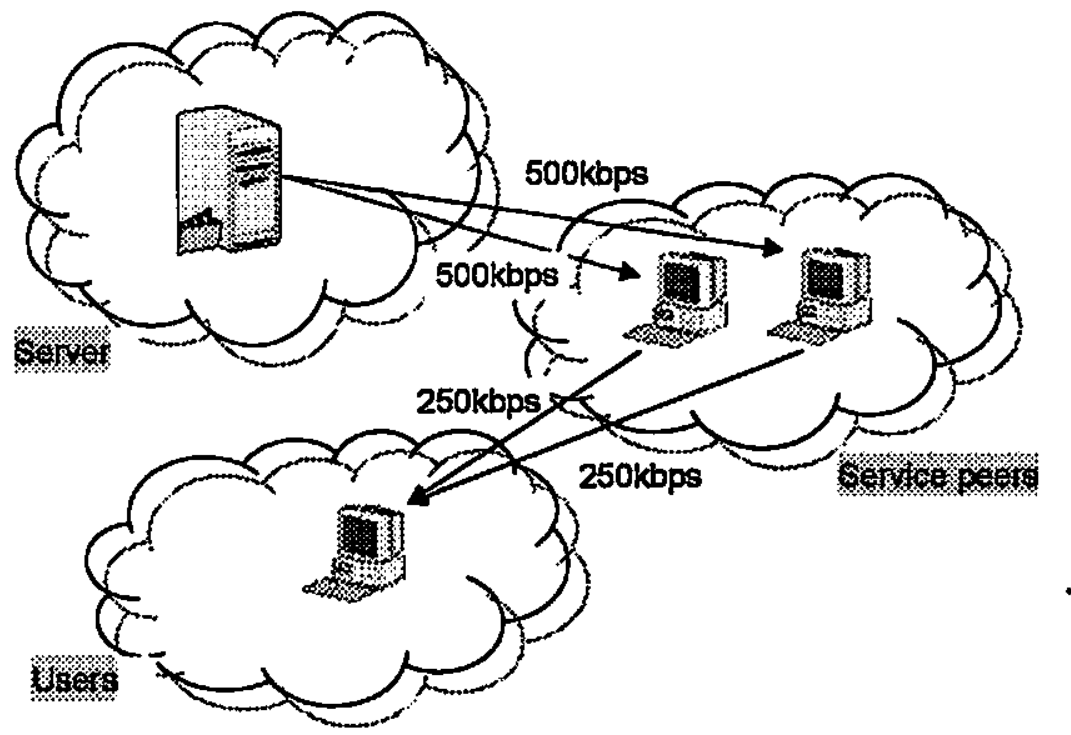

(c) Peer-to-peer Network

Figure 2: Video streaming network examples 
On the other side, a pure CDN server cluster approach is expensive to scale. Maintaining a distribution pipe big enough to support the simultaneous video streams and a persistent $500 \mathrm{kbps}$ bandwidth per stream for a temporal video length ranging from one to three hours is very expensive.

Considering the pros and cons of the CDN and P2P approach, in this thesis, we propose a hybrid system that integrates $\mathrm{CDN}$ and $\mathrm{P} 2 \mathrm{P}$ strategies. The system allows the two streaming technologies to complement each other - the high availability of CDN servers and the scalability of P2P. We also apply MD coding to encounter the limited bandwidth of service peers and the unreliable networks. In our video delivery system, we have CDN servers with the full reservoir of videos and service peers buffering a partial of one or more videos in terms of video descriptions. When a user requests one of the supporting videos, the system selects multiple service peers who have the description of the video to serve this request collaboratively. If the service peers alone do not have sufficient bandwidth to serve this request, the CDN server will join to help with streaming.

Regarding P2P or hybrid CDN/P2P video streaming technology, many schemes from different aspects have been proposed to address reliability, quality adaptation and scalability issues. In the aspect of coding, a number of MD coding algorithms have recently been proposed, which provide different tradeoffs in terms of compression performance and error resilience [4] [21]-[24]. In particular, the MD video coding system of [24][25] enables the repair of corrupted frames in a description using uncorrupted frames in the other description so that usable quality 
can be maintained even when some descriptions are afflicted by losses. In the aspect of the delivery, delivery-path-management schemes are proposed to address the reliability issue over P2P networks. For example, in Coopnet [7], the system uses a centralized tree management algorithm to construct and maintain a diverse set of delivery paths. Many incentive contribution polices [10][28][29] are also proposed to encourage peers to commit their recourses. For example, [29] proposed applying the social taxation model to peer contribution: the amount of recourse that a peer is required to contribute is based on the quality of the service that a peer receives. More contribution leads to higher quality of the service.

From a unique aspect, we investigate video placement schemes with respect to peer upstream capacities to better utilize their upstream bandwidth. A placement scheme is to decide which video descriptions that a service peer buffers. The placement scheme design has never been the main topic in this area of research. Research that comes close to the investigating placement scheme design is [9], where Y. Shen et al. considered the number of copies for each video description make and place them among the service peers in a round-robin fashion when they were mainly exploring MD coding to reduce video quality distortion. Our goal is to design a placement scheme such that we can maximally utilize the service peer upstream bandwidth in order to maximally reduce the system load on the CDN servers. We propose several effective placement solutions and further evaluate these schemes via analysis and simulation. 
In the following, we first describe the system setting in chapter II, and present the proposed placement schemes in chapter III. We further evaluate the performance of the proposed schemes in chapter I V and conclude this paper in chapter V. 


\section{CHAPTER II \\ SYSTEM OVERVIEW}

\section{II.A System Architecture}

To support large-scale on-demand streaming, we propose to improve system capacity by exploiting the limited peers' upstream bandwidth and buffer space while ensuring stable quality in streaming. Different from the approaches[4]-[10], we focus on efficient schemes for mimimizing server load and ensuring streaming quality in a two-level hybrid architecture. In this paper, we use a hybrid streaming architecture that integrates CDN and P2P networks [5][10], as shown in Figure 3. The architecture takes advantage of the high availability of CDN servers and the scalability of P2P networks. At the upper level, an overlay network (i.e., a CDN) is used to deliver videos from a central server to proxy servers, which then deliver to the lower level, a collaborative peer network, which consist of supplying peers who commit buffer space and upstream bandwidth to assist the video delivery. In such a local network, a proxy server acts as a coordinator for directing supplying peers to service incoming video-on-demand requests. Video data is pushed to supplying peers to reduce the load of the proxy server. Proxy servers provide directory service for local clients and schedule streaming sessions to deliver video data to clients with the assistance of the supplying peers. 


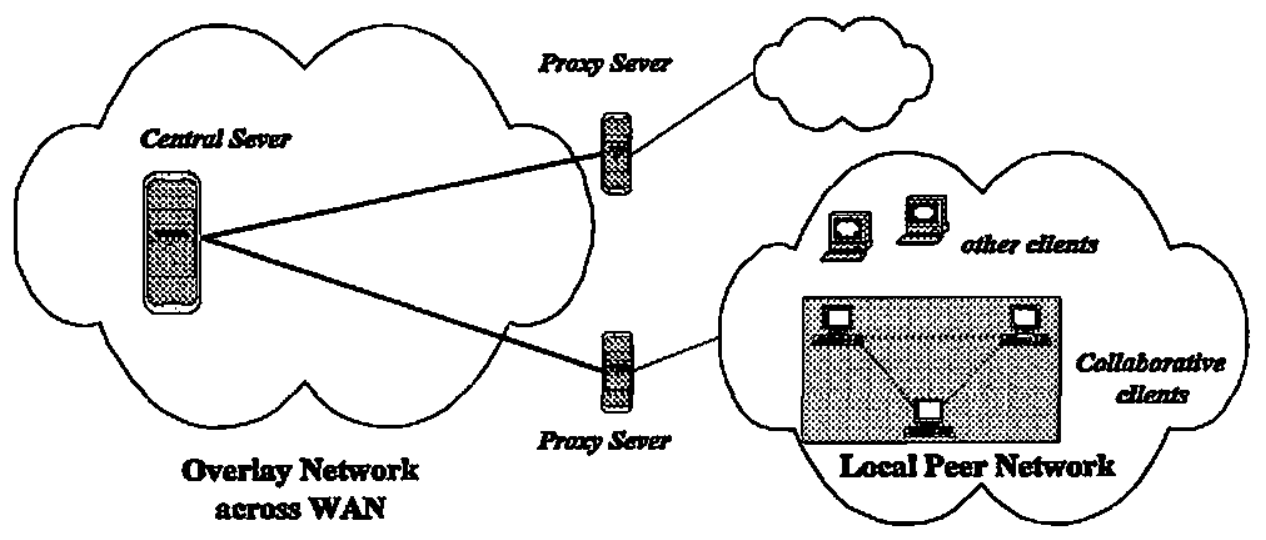

Figure 3: Hybrid Streaming Architecture

In this paper, we investigate effective placement schemes in a local collaborative network to fully exploit supplying peers' upstream bandwidth As shown in Figure 4, we have the fixed proxy server service capacity $C s$ and the potential high peer service capacity $C p$ from the supplying peers. Since a proxy usually caches much more video data than the supplying peers, we want to maximally utilize $C p$ such that $C s$ can be shared by other requests that can not be fulfilled by peers.

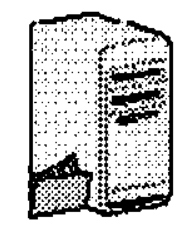

Server

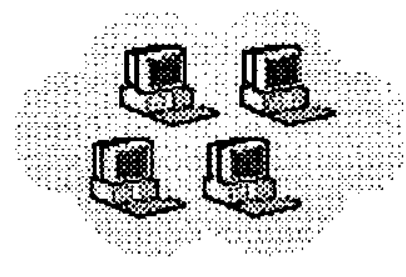

Collaborative Network

\section{Sever Service Capacity}
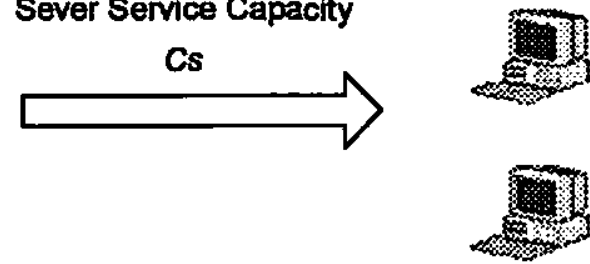

Peer Service Capacity

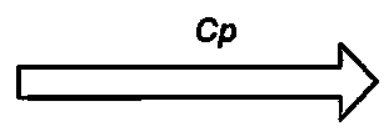

Users

Figure 4: System Architecture in a Local Network 


\section{II.B Placement Problem}

Current P2P streaming approaches mostly consider the scalability and reliability issues, e.g., the authors in [9] proposed a scheme to explore multiple-description coding to reduce quality distortion. They consider the number of copies to make and distribute them among peers in a round-robin fashion. In this thesis, we point out that a round-robin placement is not sufficient for $\mathrm{P} 2 \mathrm{P}$ networks where peers have various upstream capacities. We argue that more careful placement schemes are needed to further improve the utilization of peer upstream bandwidth. Intuitively, when data from two popular videos compete for the upstream bandwidth at a supplying peer, we observe more conflicts than between the popular video and less popular one. Figure 5 shows the importance of careful placements. Assume we have four videos with mean request rate $0.6,0.5,0.2$ and 0.1 , respectively. We have two peers with one unit of upstream bandwidth. When we do not consider the competition carefully, we may have a placement like (a), in which peer 1's upstream bandwidth may be not sufficient to service the two popular videos, while peer 2's upstream bandwidth may be under-utilized since it buffers two less popular videos. A careful placement such as case (b) may make the upstream bandwidth of both peer 1 and 2 highly utilized, so that the overall peer contribution is better than case (a). 


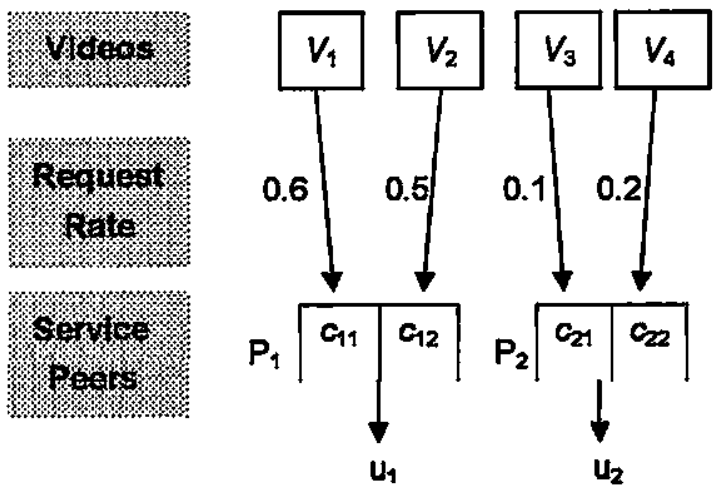

(a) Peer $P_{1}$ may be always busy, while $P_{2}$ may be always idle

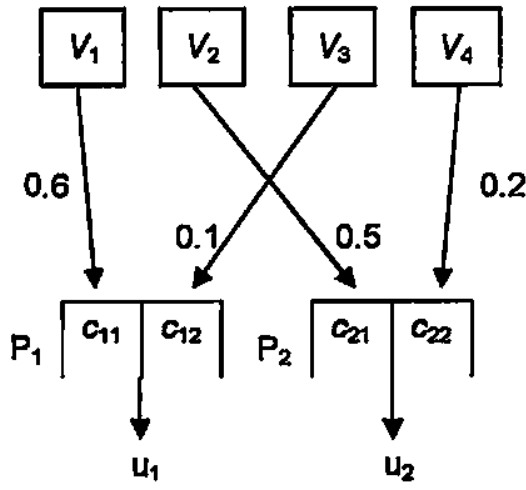

(b) Both peer $P_{1}$ and $P_{2}$ are evenly loaded

Figure 5: Examples of competition with different placements

Therefore, when we determine where to push a copy, we need to consider not only the available upstream bandwidth at a peer but also the competition of different video data on its upstream bandwidth. In the following, we focus on this issue and investigate the effective placement schemes to fully utilize peer upstream bandwidth.

\section{II.C Definitions and Assumptions}

The proposed system supports on-demand service for a fixed set of $N v$ videos and the popularities of these videos follow a Zipf distribution. We assume that we do not know arrival times as a priori; the inter-arrival times of a video $j$ following an exponential distribution with a mean rate $\lambda, 1 \geq j \geq N v$. To deal with peer dynamic and limited upstream capacities, we use a MD coding to encode the video data $\mathbf{A}$ video data is encoded into multiple descriptions and these descriptions are stored at different peers to ensure data availability. (Each description is corresponding to one buffer slot and one upstream unit at a peer.) For ease of illustration, we assume that all 
videos have the same number of descriptions and the bandwidth of a description is $U_{0}$. (To further simplify the discussion, we use one description per video in our examples.)

We have $N_{p}$ service peers in a collaborative network. Supplying peer $i$ commits a buffer space of $b_{i}$ slots and an upstream bandwidth of $u_{i}$ units for assisting a proxy server to deliver videos. Here, buffer slots are the same size, i.e., a buffer slot holds a video description; a unit can be used to deliver a video description at rate $U_{0}$ An upstream unit is used as an upstream channel to deliver a video description. We assume that we have sufficient bandwidth between peers in local networks, while the peer upstream bandwidth is the common bottleneck. Supplying peers are willing to contribute for a long period to improve its status/priority in the system, e.g, to gain the quality assurance for its future sessions.

\section{II.D Placement Problem}

We consider a video delivery system with $N_{p}$ service peers. The numbers of buffer slot $b_{1}$ and upstream unit $u_{i}$ for a peer $i$ are known. The system supports $N v$ videos. The mean request rate $\lambda_{j}$ of video $j$ is given. A placement is denoted as $\Psi=$ $\left\{c_{1,1}, \ldots, c_{1, b 1} ; c_{2,1}, \ldots, c_{2, b 2} ; \ldots ; c_{\mathrm{Np}, 1}, \ldots, c_{\mathrm{Np}, b \mathrm{~Np}}\right\}$, where $c_{1, t}$ is corresponding the video that has a copy placed at the $t$ th slot of peer $i$. For example, the placement shown in Figure $5(a)$ is $\Psi=\{1,2 ; 3,4\}$ and the placement shown in Figure 5 (b) is $\Psi=\{1,3$; $2,4\}$. Alternatively, we can view a placement as a one-to-many mapping from videos to peer buffer space, as shown in Figure 6. For instance, regarding the system shown 
in Figure 5, once we determine the number of copies for each video - in this case, we have one copy for each video, the placement is a one-to-one mapping, which is special case of one-to-many mapping. Figure 5 shows two different mappings.

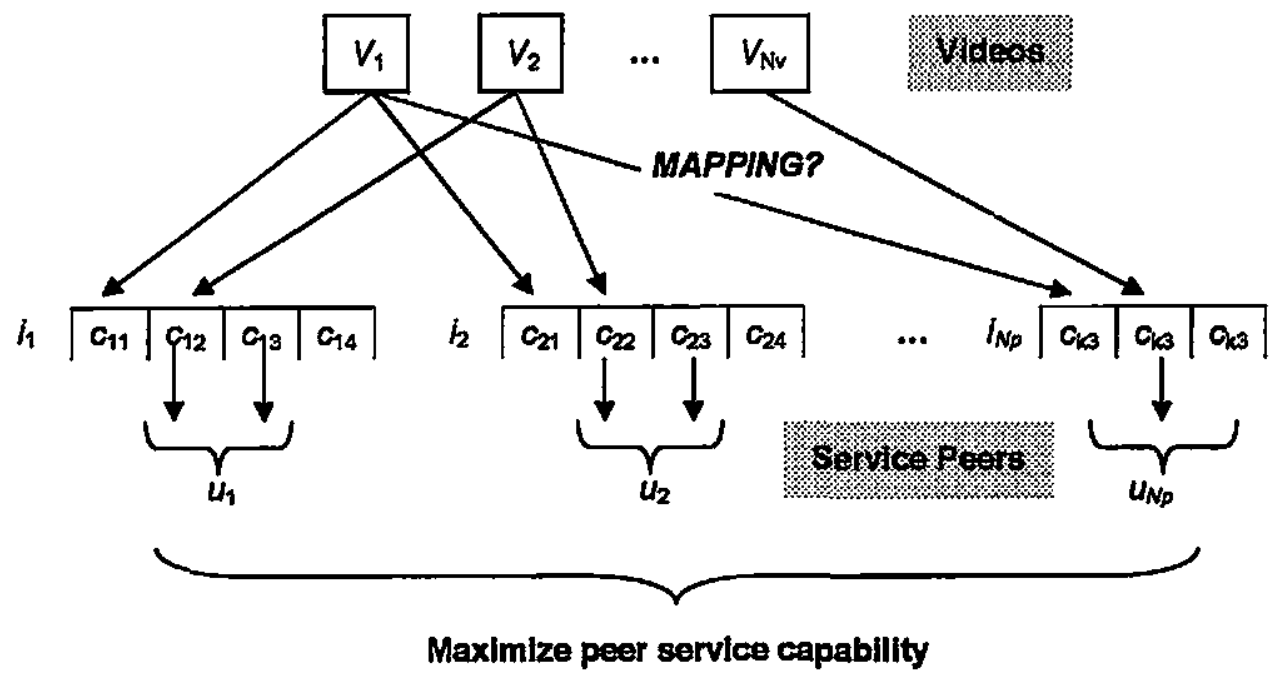

Figure 6: Placement mapping: number of copies and where to put them

Our goal is to determine the placement $\Psi$ such that the peer contribution is maximized, i.e, the utilization of peer contributed upstream bandwidth is maximized. $\max : \sum_{j=1}^{N p} \rho_{j}$

Where $\rho_{i}$ is the upstream bandwidth utilization of peer $i$ during the process of video distribution.

The following chapter will illustrate several schemes to determine the one-to-many mapping. 


\section{CHAPTER III EFFICIENT PLACEMENT SCHEMES}

In this section, we investigate efficient placement schemes for fully exploiting peer upstream bandwidth. Because we need to determine the number of copies to make and where to store them in the process of placement, we investigate the placement schemes in the following two cases to examine the efficiencies of different placement methods: 1) two-step schemes - first determine the number of copies of videos and then distribute these copies to peers; 2) a unified scheme that considers both the number copies to make and where to store them at the same time to fully exploit the peer upstream bandwidth. For the first case, we proposed a bandwidth-based placement scheme, and then compare its performance with a round-robin method [9] in various settings. For the second case, we develop a utilization-based scheme that reduces competition at peers and fully utilizes peer contributions.

\section{III.A Two-step Placement Schemes}

A two-step placement scheme first determines the number of copies for a video. In this paper, we choose the mumber of copies to make for a video proportional to its requesting rate, ie., the number of copies for video $j, C j$, is defined as

$$
C_{j}=\operatorname{round}\left(\frac{\lambda_{j}}{\sum_{k=1}^{V} \lambda_{k}} \sum_{i=1}^{N_{p}} b_{i}\right)
$$


The second step is to assign these copies to the supplying peers, e.g., in a round-robin fashion as in [9]. In this paper, we propose a bandwidth-based scheme that will be introduced in the following section.

\section{III.A.1 Round-robin placement}

A round-robin placement approach was proposed in [9] using multiple-description coding to reduce quality distortion. It used a dynamic-programming approach to determine the number of copies of each video description to create. It then sorts video copies based on their expected access rates and distributes them among peers in a round-robin fashion. The algorithm is summarized as:

1) After the number of copies of the videos $\left\{C_{l}, \ldots, C_{N v}\right\}$ is determined, sort videos in a descending order by $\lambda j$.

2) Assign an index $I(j, n)$ to the $n$th copy for video $j$. The index is calculated as follows:

$$
I(j, n)=\sum_{k=1}^{j-1} C_{k}+n
$$

3) Place the copy indexed by $I(j, n)$ on the supplying peer $I(j, n) \bmod N p$.

This algorithm assumes the peers have the same buffer space. However, it does not fit for peers with various buffer spaces: in step 3, it may tend to place a copy at a peer without any free buffer slots. To fit into the P2P settings, we modify the algorithm as follows: 
1) After the number of copies of the videos $\left\{C_{1}, \ldots, C_{\mathrm{Nv}}\right\}$ is determined, then sort the copies in descending order by $\lambda$.

2) Assign an index $I(j, n)$ to the $n$th copy for video $j$. The index is calculated as follows:

$$
I(j, n)=\sum_{k=1}^{j-1} C_{k}+n
$$

3) Assign an index $J(t, i)$ to the th buffer slot of peer $i$. The index is assigned as follows:

3.1. Create an indicator matrix $I d, \max \left\{b_{t}\right\}$ by $N p$. Assign an indicator to each cell:

$$
I d(t, i)= \begin{cases}1 & t \leq b_{i} \\ 0 & t>b_{i}\end{cases}
$$

3.2. The index is calculated as:

$$
J(t, i)=\sum_{n=1}^{t-1}\left(\sum_{k=1}^{N p} I d(n, k)\right)+\sum_{k=1}^{i} I d(t, k)
$$

4) Place the copy indexed by $I(j, n)$ on the buffer slot indexed by $J(t, i)$.

\section{III.A.2 Notations for bandwidth-based placements}

We summarize the notations used in the following with Table 1. Each upstream bandwidth unit uses one channel for delivery. Since every channel can be used by any buffered video, we have $v_{i}$ videos compete for each channel at peer $i$ with a total expected access rate $a_{l}$, in which video $j$ requests may access a channel with an access rate $r_{j}$. The total bandwidth requirement of video $j$ can be estimated by its arrival rate 


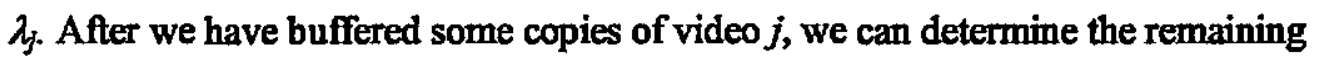
bandwidth of video $j$, denoted as $s$, to help us direct which video we should buffer next.

Table 1: Key Notations

\begin{tabular}{|c|l|}
\hline$u_{i}$ & The number of upstream channels(units) of supplying peer $i$ \\
\hline$v_{t}$ & The number of videos currently buffered at peer $i$ \\
\hline$r_{j}$ & The current access rate of video $j$ per upstream channel \\
\hline$a_{l}$ & The total access rate per upstream channel at peer $i$ \\
\hline$s_{j}$ & The remaining required upstream bandwidth of video $j$ \\
\hline
\end{tabular}

Now we show how to estimate $r_{j}$, the access rate of video $j$ per upstream channel.

We first count the total number $N_{j}$ of the upstream channels possibly accessible by video $j$ requests, which is the sum of the upstream channels of the peers who buffer a copy of video $j$, so we have

$$
N_{j}=\sum_{i=1}^{N_{p}} u_{i} \cdot \min \left\{\sum_{t=1}^{b_{i}} \eta(i, t, j), 1\right\}
$$

Where $\eta(i, t, j)$ is an indicator of whether $c_{i, t}$ is a copy of video $j$. If it is true then $\eta(i, t, j)$ is 1 , otherwise 0 .

$\eta(i, t, j)= \begin{cases}0, & c_{i, t} \neq j \\ 1, & c_{i, t}=j\end{cases}$

Assume that the incoming requests of a video are distributed to its supporting channels with equal probability, and then we have the access rate $r_{j}$ of video $j$ per upstream channel as follows: 


$$
r_{j}=\frac{\lambda_{j}}{N_{j}}
$$

After we determine $r_{j}$, we can compute the total access rate $a_{t}$ per upstream channel at peer $i$, shown in the equation as follows.

$$
a_{i}=\sum_{n=1}^{b_{i}} r_{c_{i, n}}
$$

For the ease of illustration later, denote $A=\left\{a_{1}, \ldots, a_{N p}\right\}=f_{a}(c, u, b, \lambda)$.

Now we show how to estimate the remaining required upstream bandwidth $s_{j}$ of video $j$. It is measured by the difference of the target upstream bandwidth demand $t_{j}$ of the video and the upstream bandwidth $w_{j}$ that it already has obtained. We use arrival rate $\lambda_{j}$ of video $j$ to estimate its target bandwidth requirement $t$. We want to equally ensure the acceptance rate for each video, so we split the total system upstream bandwidth into portions where each video has a piece proportional to its requesting rate. As shown in the Eq.10, the term $\sum_{i=1}^{N p} u_{t}$ is the total system upstream bandwidth.

$$
t_{j}=\left(\sum_{i=1}^{N p} u_{i}\right) \cdot \frac{\lambda_{j}}{\sum_{k=1}^{N v} \lambda_{k}}
$$

The upstream bandwidth $w_{j}$ that video $j$ already has obtained is the sum of the upstream bandwidth that each of its copy obtains.

$$
w_{j}=\sum_{i=1}^{N p}\left(\sum_{t=1}^{b_{i}} \alpha_{c_{i, t}} \cdot \eta(i, t, j)\right)
$$

Where $\alpha_{c t, t}$ is the fraction of upstream channels that copy $c_{i x}$ obtains. Consider $r_{c t, t}$ as the competition capability of each copy at peer $i$, then we have $\alpha_{c, l}$ as follows: 


$$
\alpha_{c_{i, 5}}=u_{i} \frac{r_{c_{i, s}}}{\sum_{n=1}^{b_{i}} r_{c_{i, t}}}
$$

Hence, we now have the remaining required upstream bandwidth $s j$ of video $j$.

$$
s_{j}=t_{j}-w_{j}
$$

For the ease of illustration later, denote $S=\left\{s_{1}, \ldots, s_{N v}\right\}=f_{s}(c, u, b, \lambda)$.

\section{III.A.3 Bandwidth-based placement}

As storage space becomes much cheaper, the upstream bandwidth will be the key limitation in peer contribution. We have found that, when peer buffer space is beyond a certain size, increasing the buffer size of a peer usually helps very little in improving its contribution, because of its limited upstream bandwidth.

Because a round-robin placement does not consider the upstream capacities of peers, it is not fit for peers with various upstream capacities. Therefore, we develop several placement schemes that exploit the peer upstream differences to improve their utilization in the following.

We first design a bandwidth-based placement that considers both the access rates of different video copies at peers and the differences between upstream capacities of peers. The basic idea is to evenly distribute requesting bandwidth among peers with respect to their upstream capacities. To achieve this, we first divide peers into classes based on their upstream capacities, because we usually have only several types of peers, e.g., connected through DSL/Cable modem or Ethernet. Within a class, we 
allocate buffer slots at peers to cache videos proportional to their request rates.

To process a class, we first sort peers based on their current buffer capacities; for the peers with the same buffer capacity, we sort them based on their current access rates (i.e., their current loads). We then allocate videos to the sorted peers, one-by-one from high to low, based on their remaining bandwidth requirements. Starting from video $j$, we allocate $C j$ copies to the sorted peers in the class, where $C j$ is determined by the number of copies for each video in this class based on their expected arrival rates, $\lambda, 1 \geq j \geq N v$, and the total buffer capacity of peers in this class as introduced above. Repeat the process until all copies of the videos are assigned or all buffers of these peers are allocated.

The bandwidth-based placement scheme is summarized as follows:

1) Divide the peers into classes; each peer in the same class has same number of buffer slots and upstream channels.

2) Sort the class with the primary key, which is the total buffer space of the class in ascending order and with the secondary key, which is the number of upstream channels in ascending order.

3) For each class in the sorted order

3.1 Given the total number of buffer slots of the class, compute the number $C_{1} \ldots C_{N v}$ of copies based on their request rate,.

3.2 Select the unassigned video $j$ with the highest $s$.

3.3 Sort the peers in this class by $a$.

3.4 Place the $C_{j}$ to the sorted peers. 
3.5 Repeat step 3.2 until all videos have their copies assigned

We choose to process the classes from the one with the lowest upstream capacities to the highest. The key reason is that the high-upstream classes provide us room to adjust the placement in the later rounds. For the same reason, in a class, we start from the peers with the fewest number of buffer slots.

\section{III.B Utilization-based placement}

The basic idea is to select a video with the highest remaining bandwidth requirement and assign a copy of this video to the supplying peer with the most available upstream bandwidth. Since the accesses of peers are dependent on each other because they may partially buffer the same data, the utilization of a peer is varied in the process of placement and is not stable until all video copies are allocated. Therefore, although the immediate estimation of peer upstream utilization helps us to determine placement, we can not totally depend on it to achieve efficient placement. To address this issue, we use $u_{i}, c_{l}$ and $a_{l}$ to estimate which peer has the more available upstream bandwidth, based on the following rules:

(1) When the number of the buffered copies at one peer is more than that of another peer, the former peer tends to have higher utilization per channel.

(2) When two peers buffer the same number of video copies while they have different upstream capacities, the one with higher upstream capacity statistically has more available upstream bandwidth

(3) When two peers buffer the same number of video copies and they have the same 
upstream capacity, the one with a lower access rate has more available upstream bandwidth.

Our utilization-based placement scheme is as follows:

1. Select video $j$ with the highest remaining bandwidth $s$.

2. Select peer $i$ from peers with available buffer slots but without a copy of video $j$.

We first choose peer $i$ from these peers with the highest upstream capacity; among the peers with the same upstream capacity, we choose the peer with the fewer number of videos; if we still have a tie, we choose the peer with the lowest access rate.

3. If peer $i$ is not found, select another video with the next highest $s$ and repeat step 2; otherwise, we place a copy of video $j$ to peer $i$.

4. Repeat step 1 to 3, until all buffer slots at peers are allocated with video copies.

\section{III.C Placement Adjustment Scheme}

The proposed placement schemes do not always yields a placement where the upstream bandwidth is evenly distributed to the videos with respect to their requesting rates. At the end the placement scheme, we may not be able to place a copy of a video with the highest remaining bandwidth requirement $s$ to a peer with the most available upstream bandwidth, because the peer already has a copy of that video. The worse case is that all the peers with free buffer slots have a copy of this video. In consequence, this video cannot assign more bandwidth and furthermore, loses some bandwidth due to different videos competing with it. Although this may affect the 
placement performance in a network with a small number of peers, it generally has little impact when the number of peers is large. In the meantime, we propose the following adjustment scheme to deal with such boundary cases. The basic idea of the adjustment scheme is to replace a buffered copy or switch copies between peers to balance the remaining bandwidth requirement among videos, i.e., making the remaining bandwidth requirements of videos similar. First, we try to replace a copy of the video with the lowest $s$ with a copy of the video with the highest $s$. The next strategy is to exchange between a copy of the video $j$ with the lowest $s$ from one peer, which this copy can compete for more bandwidth and a copy of a video with the highest $s$ from another peer, which this copy can only compete for less bandwidth Then, we reevaluate how evenly the bandwidth is distributed after these adjustments to determine if such adjustments are necessary.

One way to measure how evenly the bandwidth is distributed to the videos is by their remaining required bandwidth $s$. Recall that a video with positive $s$ means it needs more bandwidth to reach its target required bandwidth. A video with negative $s$ means it has extra bandwidth As a result, we need to bring $s$ close to zero as much as possible for each video in order to have a placement with bandwidth evenly distributed.

The replacement adjustment is to replace a copy of the video $j$ containing the lowest $s$ with a copy of the video $k$ containing the highest $s$. Among the peers with a copy of video $j$ but without a copy of video $k$, we want to find the one who allows the copy of video $k$ to compete with more bandwidth. The peer with fewer buffer slots 
and lower total access rate per upstream channel is the best choice.

The exchange adjustment is to exchange between a copy of the video $j$ with the lowest $s$ from one peer where this copy competes with a large amount of bandwidth and a copy of the video $k$ with the highest $s$ from another peer, which this copy competes with a little amount of bandwidth. The peer, who has a copy of video $k$. higher $a$ and fewer $u$, tends to make this copy compete with less bandwidth. On the other side, the peer, who has a copy of video $j$, lower $s$ and more $u$, make this copy competitive with more bandwidth

In each iteration of the adjustment scheme, it does replacement and exchange adjustment separately. Then for each of the two resulting placements, compute the sum $d$ of the absolute value of $s$ of all videos, shown as the Eq. 13. If the smaller sum is also smaller than the one for the previous placement, then we accept the corresponding adjustment, otherwise the iteration stops and the previous placement is the best one we can get.

$$
d=\sum_{j=1}^{N v}\left|s_{j}\right|
$$

\section{III.D Placement Scheme Considering Reliability of Supplying Peers}

One of the characteristics of peers in P2P networks is that the peers and their comnections are unreliable. Although we assume that the supplying peers are willing to contribute their resources for a long period and in return, they have higher priority in the system, peer resources are beyond the control of the central scheduler. The condition of access to the supplying peers is dynamic. A peer may drop off at any 
time for reasons such as power supply issues, system software instability, or network difficulties.

Here we address how to modify the proposed placement schemes when given the supplying peer reliability- the probability that the peer is connected. We denote the probability as $g$ for peer $i$.

We suggest modifying three places in the original placement schemes. One is the total number $N_{j}$ of the upstream channels possibly accessible by video $j$ requests, because the peers upstream channels are not always available. The statistical number of the upstream channels of peer $i$ that is possibly accessible should be $u_{i} g_{i}$. Therefore, Eq. 6 should be modified into

$$
N_{j}=\sum_{i=1}^{N_{p}} u_{i} \cdot g_{i} \cdot \min \left\langle\sum_{t=1}^{b_{i}} \eta(i, t, j), 1\right\}
$$

The other one is the fraction $\alpha_{c t, t}$ of upstream chamnels that copy $c_{i s t}$ obtains. For the same reason, we modify Eq. 12 as follows:

$$
\alpha_{c_{i,}}=u_{i} \cdot g_{i} \cdot \frac{r_{c_{i, t}}}{\sum_{n=1}^{b_{i}} r_{c_{i, t}}}
$$

The last place is how we sort the peer with the most remaining upstream bandwidth. If a peer has a poor reliability, even though it has a great amount of upstream bandwidth, it still may not be the one that has the most remaining bandwidth, because its resources are not always accessible. Therefore, when we sort the peer with the use of $u_{i}$ and $a_{i}$, we should modify them into $u_{i} g_{i}$ and $a_{i} g_{i}$ respectively. 


\section{CHAPTER IV}

\section{PERFORMANCE EVALUATION}

\section{IV.A Analysis of Proposed Schemes}

We first present the analysis of utilization and a numerical example to show that given a fixed number of copies for each video, different placements yield different utilizations. For example, a collaborative network consists of two supplying peers, $\mathrm{X}$ and $\mathrm{Y}$ as shown in Figure 7. $\mathrm{X}$ has two buffer slots and one upstream channel and $\mathrm{Y}$ has three buffer slots and two upstream channels. The network supports the distribution of four videos, $A, B, C$ and $D$ with the average rate of $\lambda_{A}, \lambda_{B}, \lambda_{C}$ and $\lambda_{D}$ per one time unit, respectively. The videos have the same video length $1 / \mu$ time unit. Given a placement where peer $X$ buffers video $A$ and $B$ and peer $Y$ buffers video $A, C$ and $\mathrm{D}$. We assume that, when a request of a video arrives and multiple peers have a free upstream channel to support this request, we uniformly assign a peer to service this request.

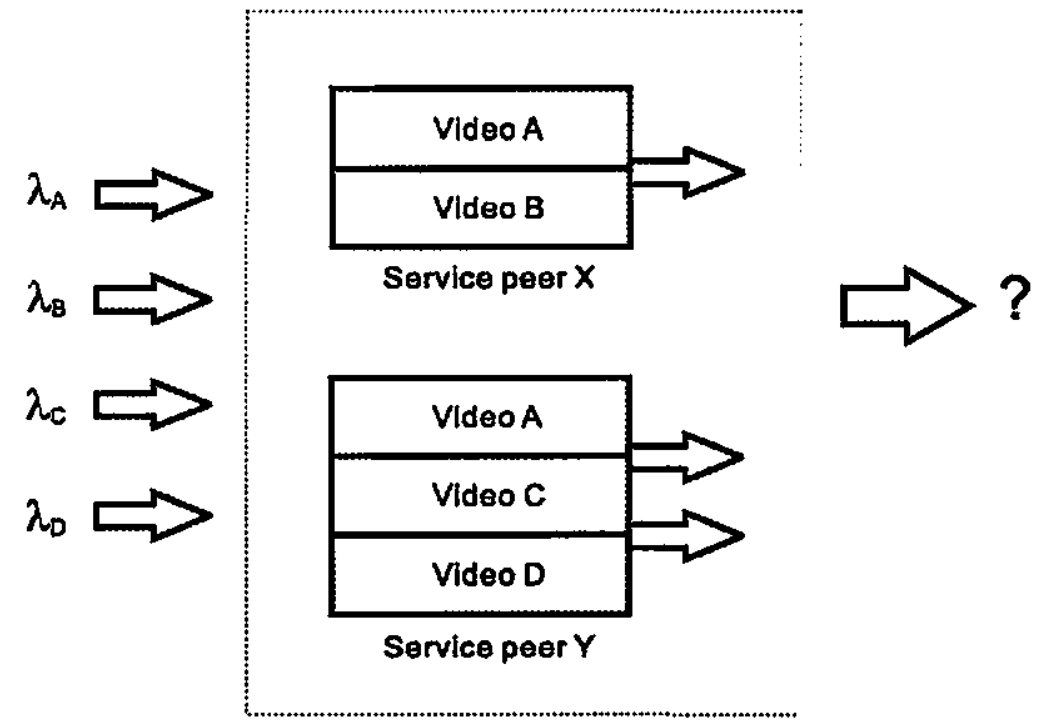

Figure 7: An example of a collaborative network 
The solution can be derived by describing the system as a 2D birth-and-death process. Denote $\mathrm{X}(s) \mathrm{Y}(t)$ as a state in the system when $s$ upstream channels of $\mathrm{X}$ are allocated and $t$ upstream channels of $Y$ are allocated. The system is operating in a total of six states, as shown in Figure 8. We index the six states from 0 to 5 in the sequence of $X(0) Y(0), X(0) Y(1), X(0) Y(2), X(1) Y(0), X(1) Y(1)$ and $X(1) Y(2)$. Then the transition rate diagram can be described by a transition rate matrix $M . M(s, t)$ is the transition rate from the state indexed by $s$ to the state indexed by $t$. For state $\mathrm{X}(0) \mathrm{Y}(0)$, the upstream channels in both $\mathrm{X}$ and $\mathrm{Y}$ are free. Its transition rate to state $\mathrm{X}(1) \mathrm{Y}(0)$ is $\lambda_{\mathrm{A}} / 2+\lambda_{\mathrm{B}}$, and to state $\mathrm{X}(0) \mathrm{Y}(1)$ is $\lambda_{\mathrm{A}} / 2+\lambda_{\mathrm{C}}+\lambda_{\mathrm{D}}$, because the request rate of video $A$ is equally distributed within the service $X$ and $Y$. We can derive the rest of the transition rates in the same manner. Finally we can obtain the transition rate matrix M shown in Eq. 16.

$$
M=\left[\begin{array}{llllll}
0 & \lambda_{A} / 2+\lambda_{C}+\lambda_{D} & 0 & \lambda_{A} / 2+\lambda_{B} & 0 & 0 \\
\mu & 0 & \lambda_{A} / 2+\lambda_{C}+\lambda_{D} & 0 & \lambda_{A} / 2+\lambda_{B} & 0 \\
0 & 2 \mu & 0 & 0 & 0 & \lambda_{A}+\lambda_{B} \\
\mu & 0 & 0 & 0 & \lambda_{A}+\lambda_{C}+\lambda_{D} & 0 \\
0 & \mu & 0 & \mu & 0 & \lambda_{A}+\lambda_{C}+\lambda_{D} \\
0 & 0 & \mu & 0 & 2 \mu & 0
\end{array}\right]
$$

(Eq. 17) 


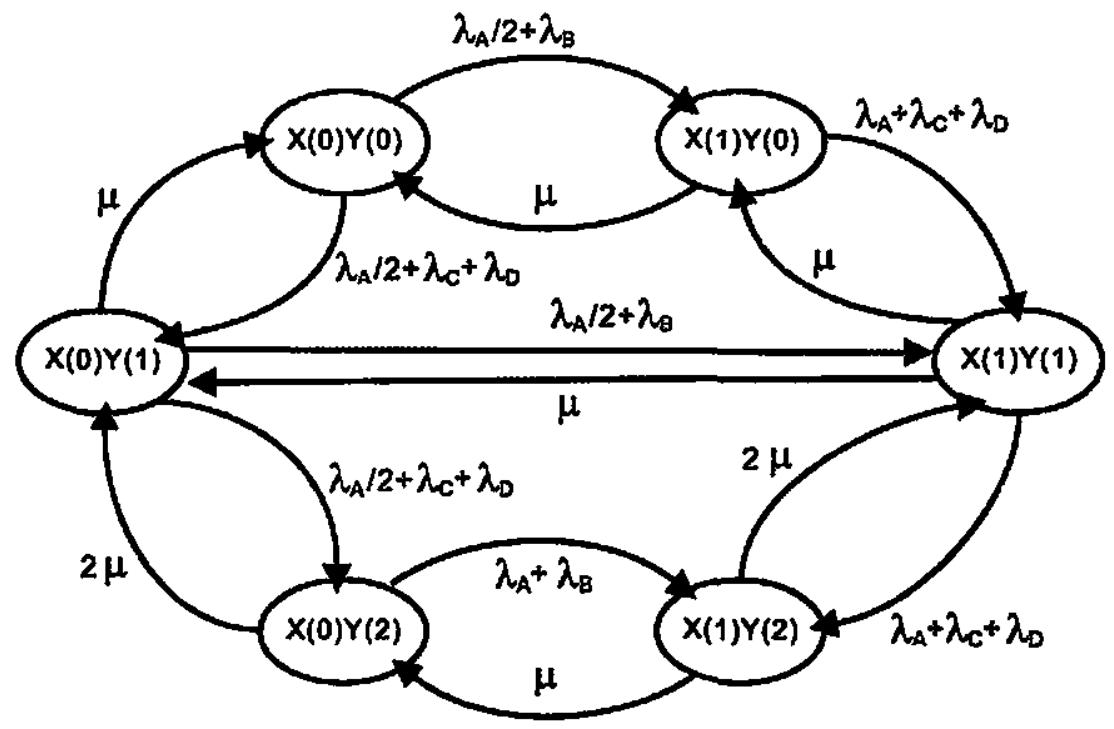

Figure 8: State-transition diagram for the example

When the system reaches a steady state, the mean transition rate into the state is equal to the mean transition rate out of it for each state. We denote the steady state probability of state $\mathrm{k}$ as $P_{k}$. We then have six balanced equations to express the equilibrium condition. However, the set of these six equations has a rank of five but has six unknowns. We need one more equation in order to solve for the unknowns. Since the system must operate in one of these states, the sum of $P_{k}$ is equal to one, which is the sixth equation. The final set of linear equations is shown in the following.

$$
\left\{\begin{array}{l}
\forall k \in\{0, \ldots, 5\}, \quad P_{k} \sum_{j \in\{0, . ., 5\}} M_{k, j}=\sum_{i \in\{0, . ., 5\}, i \neq k} P_{i} M_{i, k} \\
\sum_{k \in\{0, . ., 5\}} P_{k}=1
\end{array}\right.
$$

We solve these equations for $P_{0}, \ldots, P_{3}$. For a state, if it has $n$ out of three upstream channels are allocated, the utilization for the time in this state is $n / 3$. Therefore, the utilization of the whole system is:

$\rho=\frac{1}{3} P_{1}+\frac{2}{3} P_{2}+\frac{1}{3} P_{3}+\frac{2}{3} P_{4}+P_{5}$ 
For example, video $\mathrm{A}, \mathrm{B}, \mathrm{C}$ and $\mathrm{D}$ have the request rate $1.3,1.2,0.4$ and 0.1 per time unit respectively. Given the placement shown in Figure 7, where $\mathrm{X}$ buffers video A, B and $Y$ buffers video A, C, D, we obtain $M$ and identify steady state probability $P_{k}$. We then have the system utilization of $57.6 \%$.

Now consider another placement, which has the same number of copies for each video, in which $X$ buffers video $A$ and $D$ and $Y$ buffers video $A, B$ and $C$. We have a better utilization of $61.2 \%$. This clearly shows, when the number of copies for each video is fixed, different placements have different utilization. The key reason of such a difference is due to the limited upstream bandwidth of peers.

We further generalize this analysis for a collaborative network with given peer capacities to support a fixed number of videos. Although this model is able to find the optimal placement for maximum system utilization through exhaustive searching, the computation overhead is extremely high. Therefore, we further develop the following analysis model to estimate the system utilization.

We compare the analytical model with simulation results to verify the accuracy of the analysis. For a given placement, we first obtain the utilization based on the above analysis. We then run a simulation over 1000 time units and measure the actual upstream utilization, (detailed procedure is presented in Table 3). As shown in Table 2, the differences between the utilization from analysis and the measured utilization in simulations are negligibly small In this comparison, the system setting is as follows: we have $75 \%$ of class- 1 peers and $25 \%$ class- 2 peers. We have two testing configurations: (1) a class-1 peer has one upstream chamnel and three buffer slots; a 
class-2 peer has three upstream chamnels and three buffer slots. (2) a class-1 peer has one upstream channel and two buffer slots; a class-2 peer has three upstream channels and three buffer slots.

Table 2: Analytical vs. Simulations

\begin{tabular}{|c|c|c|c|}
\hline Configuration & & Average difference \% & Max difference \% \\
\hline \multirow{3}{*}{1} & Utilization-based & 0.05 & 0.07 \\
\cline { 2 - 4 } & Bandwidth-based & 0.04 & 0.09 \\
\cline { 2 - 4 } & Round-robin & 0.05 & 0.09 \\
\hline \multirow{3}{*}{2} & Utilization-based & 0.03 & 0.06 \\
\cline { 2 - 4 } & Bandwidth-based & 0.04 & 0.09 \\
\cline { 2 - 4 } & Round-robin & 0.03 & 0.04 \\
\hline
\end{tabular}

Figure 9 shows the differences between three placement schemes. For a small scale system with six videos and eight to twenty peers, the proposed approaches have clear advantages over the round-robin scheme.

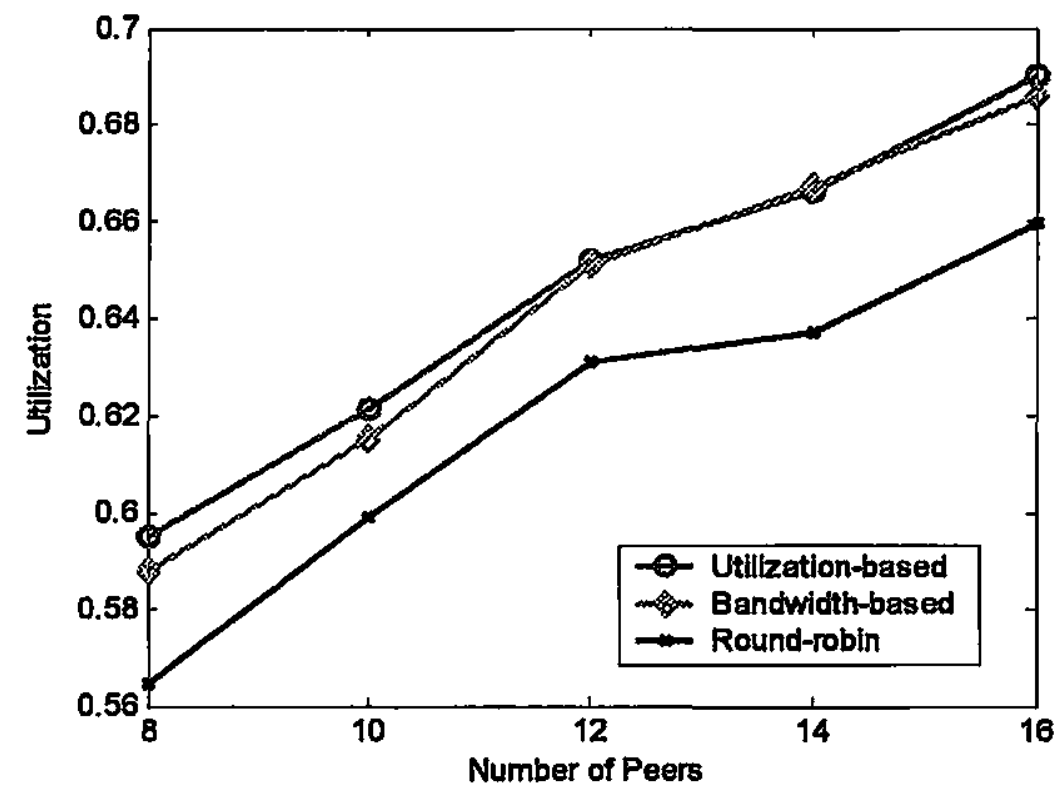

Figure 9: Analytical comparison of the utilization of three placements 


\section{IV.B Simulation}

In this session, we use simulation to evaluate the proposed schemes from various aspects. We first examine the proposed placement schemes, then the adjustment scheme, the effect of the system load, and finally the consideration of the peers' reliability. Table 3 shows the procedure we use to measure the utilization of the system when given a placement.

Table 3: Pseudocode for the simulation to measure the utilization of the system Input: $\quad U[1, N p]$ : array of the upstream bandwidth for each peer $B[1, N p]$ : array of the buffer size for each peer $\pi 1, N v]$ : array of the arrival rate for each video $\Psi[1, N p][1, \max (b)]$ : placement, a matrix of the video copies buffered at each slot of each peer

Output: $\quad P$. utilization

Parameter: $\quad \operatorname{mark}[N p, \max (B)]$ : mark the time that a channel of a peer is available duration: length of the simulation count: count total time unit that any channel in the system is used

PlacementUtilization $(U, B, 7, \Psi)$ generates a sequence of requests based on the arrival rate of the videos for a certain period of time, then simulates the operation of the distribution system to service these requests and counts the time length of each upstream channel used. At the end, it calculates the overall utilization of all channels.

1. $\triangleright$ Initialization

2. for each video $j$, next_arrival_time $\left.[j] \leftarrow f_{\text {interval }}(\pi j]\right)^{*}$

3. [cur_arrival, cur_j] $\leftarrow \min ($ next_arrival_time)

4. for each peer $i$, for each channel $n, f l a g[i, n] \leftarrow 0$

$f_{\text {mateval }}(\lambda)=-\log (1-$ rand $) / \lambda$ 
5. $D$ Iteration

6. while cur_arrival < duration

7. $S \leftarrow \varnothing$

8. for each peer $i$

9. If find $\left(\Psi[i,:]=c u r_{j}\right)$ and find(mark $[i,:]<c u r_{-}$arrival)

10. $S \leftarrow S \chi i$

11. if $S \varnothing$

12. randomly pick an element $i$ from $S$

13. $\quad$ set $n \leftarrow$ find(mark $[i,:]<c u r_{-}$arrival)

14. $\quad$ mark $[i,:] \leftarrow$ cur_arrival +1

15. count $\leftarrow$ count +1

16. next_arrival_time $\left[\right.$ cur_j] $\left.\leftarrow f_{\text {interval }}(\pi c u r j]\right)$

17. $\quad\left[c u r_{\text {_arrival, cur_j] }} \leftarrow \min (\right.$ next_arrival_time)

18. $\triangleright$ Computer utilization

19. $P \leftarrow$ count / ( $\operatorname{sum}(B) \times$ duration $)$

20. return $P$

\section{IV.B.1 Evaluation of the Proposed Placement Schemes}

Here we examine the performance advantages of the utilization-based and bandwidth-based placement schemes over the round-robin approach. Table 4 shows six simulation settings with different peer upstream bandwidth and buffer capacities used in our evaluation. For each of these settings, we further choose three different system configurations with different number of videos $N v$ and number of peers $N p$ in the system. In configuration 1 , we keep the ratio of $N p / N v$ as two and increase $N v$ from 12 to 96 . In the second set of configuration, we keep $N v$ as 20 , and increase $N p$ from 20 to 580 . In the third set of configuration, we keep $N p$ a 160 and increase $N v$ 
from 20 to 160.

Table 4: Simulation setting for comparing different placements

\begin{tabular}{|c|c|c|c|c|c|}
\hline & & & $\begin{array}{l}\text { Class } 1 \\
(50 \%)\end{array}$ & $\begin{array}{l}\text { Class } 2 \\
(25 \%)\end{array}$ & $\begin{array}{l}\text { Class } 3 \\
(25 \%)\end{array}$ \\
\hline \multirow[t]{6}{*}{ Group 1} & \multirow[t]{2}{*}{ Set 1} & $\begin{array}{c}\text { Upstream } \\
\text { bandwidth (u) }\end{array}$ & 1 & 2 & 3 \\
\hline & & Buffer size (b) & 3 & 3 & 3 \\
\hline & \multirow[t]{2}{*}{ Set 2} & $\mathfrak{u}$ & 1 & 2 & 3 \\
\hline & & b & 8 & 8 & 8 \\
\hline & \multirow[t]{2}{*}{ Set 3} & $\mathbf{u}$ & 1 & 2 & 3 \\
\hline & & b & 16 & 16 & 16 \\
\hline \multirow[t]{4}{*}{ Group 2} & \multirow[t]{2}{*}{ Set 1} & $\mathbf{u}$ & 2 & 2 & 2 \\
\hline & & b & 2 & 4 & 8 \\
\hline & \multirow[t]{2}{*}{ Set 2} & $\mathbf{u}$ & 2 & 2 & 2 \\
\hline & & $\mathbf{b}$ & 4 & 8 & 16 \\
\hline \multirow[t]{2}{*}{ Group 3} & \multirow[t]{2}{*}{ Set 1} & $\mathfrak{u}$ & 2 & 3 & 4 \\
\hline & & b & 4 & 6 & 8 \\
\hline
\end{tabular}

Table 5 shows the results of utilization-based vs. round-robin and Table 6 shows the results of bandwidth-based vs. round-robin. Both schemes outperform the round-robin scheme. Figure 10 shows a sample result from Setting 1 . For more results please see Appendix B. 
Table 5: Difierence between utilization-based and round-robin in percentages

\begin{tabular}{|c|c|c|c|c|c|}
\hline & & Configuration & Average & Max & Min \\
\hline \multirow[t]{9}{*}{ Group 1} & \multirow[t]{3}{*}{ Set 1} & 1 & 7.42 & 9.08 & 2.99 \\
\hline & & 2 & 8.43 & 10.85 & 4.78 \\
\hline & & 3 & 8.59 & 10.40 & 6.76 \\
\hline & \multirow[t]{3}{*}{ Set 2} & 1 & 3.58 & 5.41 & 1.21 \\
\hline & & 2 & 1.16 & 1.85 & 0.65 \\
\hline & & 3 & 4.66 & 6.86 & 1.22 \\
\hline & \multirow[t]{3}{*}{ Set 3} & 1 & 2.30 & 3.49 & 0.75 \\
\hline & & 2 & 0.42 & 1.46 & 0.01 \\
\hline & & 3 & 2.47 & 4.21 & 0.34 \\
\hline \multirow[t]{6}{*}{ Group 2} & \multirow[t]{3}{*}{ Set 1} & 1 & 10.69 & 12.73 & 7.67 \\
\hline & & 2 & 8.75 & 10.60 & 4.67 \\
\hline & & 3 & 11.10 & 14.17 & 9.41 \\
\hline & \multirow[t]{3}{*}{ Set 2} & 1 & 3.86 & 4.57 & 3.21 \\
\hline & & 2 & 5.07 & 5.65 & 4.18 \\
\hline & & 3 & 3.82 & 5.66 & 2.37 \\
\hline \multirow[t]{3}{*}{ Group 3} & \multirow[t]{3}{*}{ Set 1} & 1 & 0.52 & 0.77 & 0.16 \\
\hline & & 2 & 0.30 & 0.74 & 0.01 \\
\hline & & 3 & 0.23 & 0.46 & 0.03 \\
\hline
\end{tabular}


Table 6: Difference between bandwidth-based and round-robin in percentages

\begin{tabular}{|c|c|c|c|c|c|}
\hline & & Configuration & Average & $\operatorname{Max}$ & $\operatorname{Min}$ \\
\hline \multirow[t]{9}{*}{ Group 1} & \multirow[t]{3}{*}{ Set 1} & 1 & 6.43 & 7.79 & 3.06 \\
\hline & & 2 & 7.79 & 10.43 & 2.95 \\
\hline & & 3 & 7.45 & 9.47 & 5.29 \\
\hline & \multirow[t]{3}{*}{ Set 2} & 1 & 3.74 & 5.65 & 1.25 \\
\hline & & 2 & 1.09 & 1.84 & 0.56 \\
\hline & & 3 & 4.82 & 6.98 & 1.12 \\
\hline & \multirow[t]{3}{*}{ Set 3} & 1 & 2.63 & 4.32 & 1.00 \\
\hline & & 2 & 0.36 & 1.13 & 0.02 \\
\hline & & 3 & 2.90 & 4.89 & 0.29 \\
\hline \multirow[t]{6}{*}{ Group 2} & \multirow[t]{3}{*}{ Set 1} & 1 & 10.32 & 12.05 & 6.56 \\
\hline & & 2 & 8.98 & 10.82 & 3.84 \\
\hline & & 3 & 10.67 & 13.58 & 7.81 \\
\hline & \multirow[t]{3}{*}{ Set 2} & 1 & 4.34 & 5.87 & 3.30 \\
\hline & & 2 & 4.90 & 5.37 & 3.86 \\
\hline & & 3 & 4.62 & 5.53 & 3.63 \\
\hline \multirow[t]{3}{*}{ Group 3} & \multirow[t]{3}{*}{ Set 1} & 1 & 0.22 & 0.53 & 0.12 \\
\hline & & 2 & 0.27 & 0.70 & 0.10 \\
\hline & & 3 & 0.09 & 0.30 & 0.04 \\
\hline
\end{tabular}




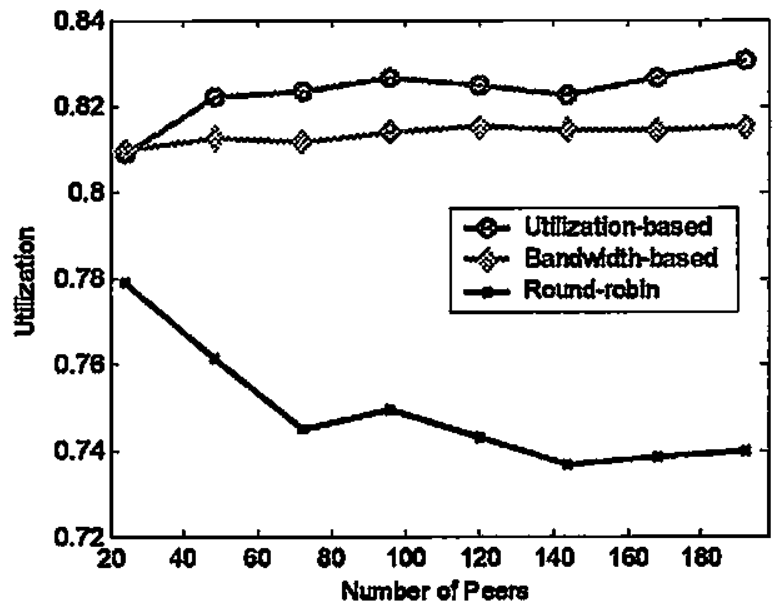

(a) Simulation results, group 1, set 1 , configuration 1

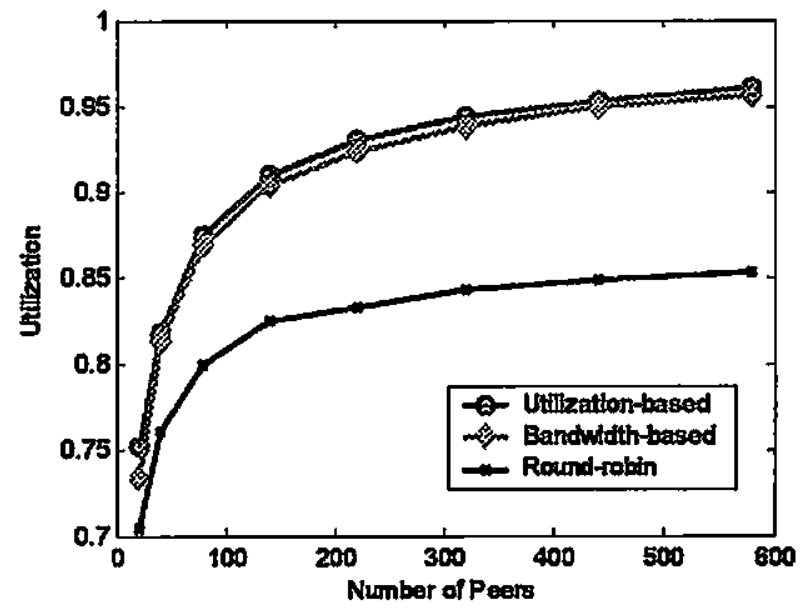

(b) Simulation results, group 1 , set 1 , configuration 2

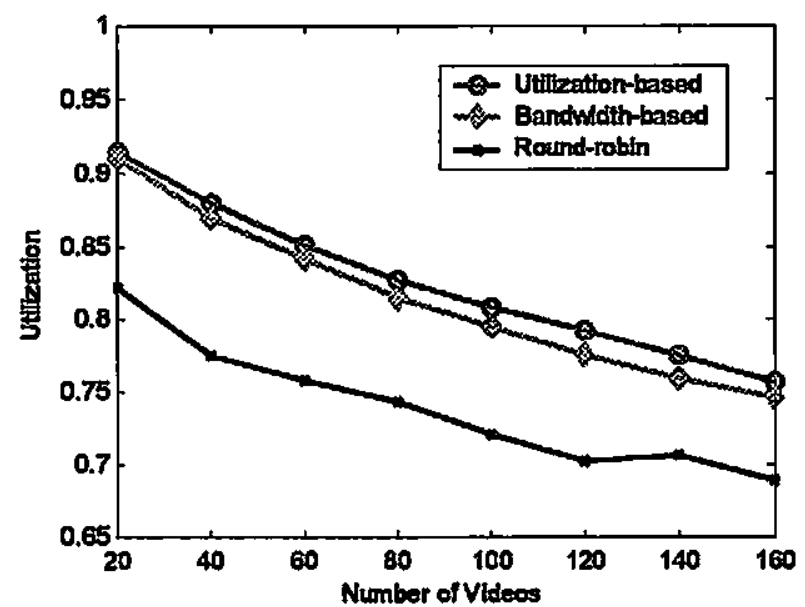

(c) Simulation results, group 1, set 1 , configuration 3

Figure 10: Sample simulation results 
In configuration 1 we keep the ratio $N v / N p$ constant, even when $N v, N p$ increase, the number of copies of each video stays the same or close to the same. In this case, the larger the system is, the more aggregated effect of each copy. Therefore, we see more advantages of utilization-based and bandwidth-based placements over round-robin ones, as shown in Figure 10 (a) and Figure 11.

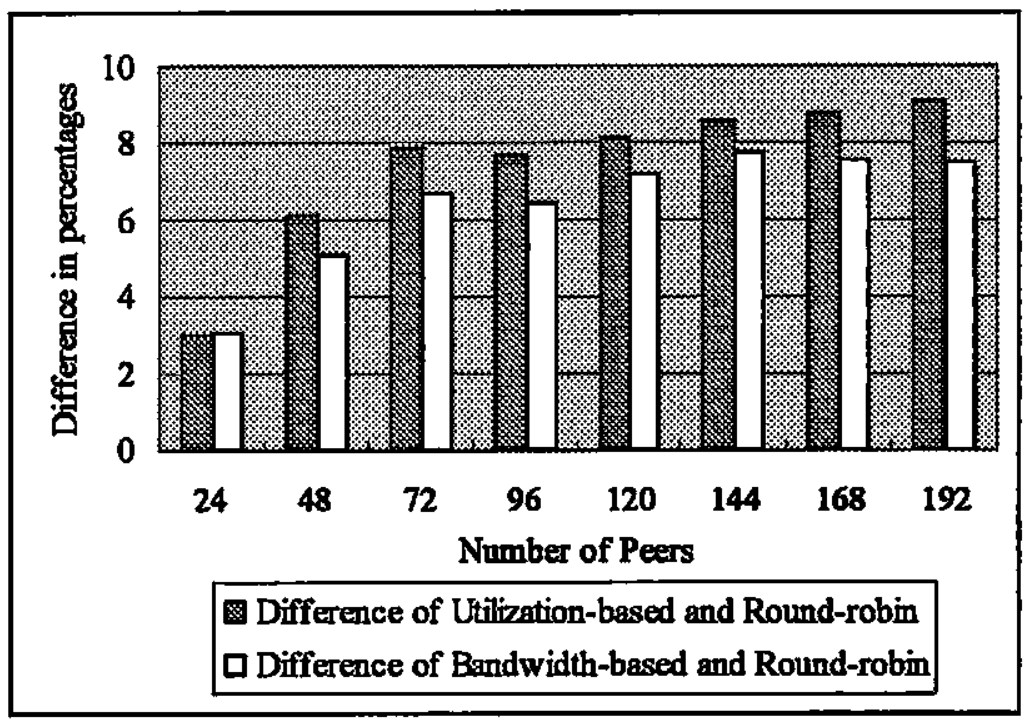

Figure 11: Difference of utilization-based/bandwidth-based and round-robin in group 1 , set 1, configuration 1

In configuration 2, we keep the number of videos constant and increase the number of peers. Figure 10 (b) shows that the utilization of the utilization-based or bandwidth-based grows faster than that of round-robin, as the number of peers increases.

In configuration 3, we keep the number of peers constant and increase the number of videos. As we see in Figure 10 (c), the utilization of the three placements drops, but still both the utilization-based and bandwidth-based keeps same amount of the percentage of the utilization higher than round-robin. 
Now we examine the three sets of experiments in group 1. In these three sets, the setup for the upstream bandwidth of the peers is the same, but the buffer size is 3,8 and 16 respectively. Both Table 5 and Table 6 show the advantages of utilization-based and bandwidth-based over round-robin decrease as the buffer size is increased. For example, the average difference between utilization-base and round-robin decreases from $7.42 \%$ to $2.30 \%$ in configuration 1 . This is because the larger the buffer size is, the higher opportunities to utilize the upstream channels and more room to improve the low end round-robin placement. This is also the same reason for the effect that we see in the group 2.

\section{IV.B.2 Evaluation of the Placement Adjustment Scheme}

Here we evaluate the performance of the placement adjustment scheme and determine the situation that it can play a role in. In the experimental setup, we have a system with a supplying peer network where $50 \%$ have one upstream channel, $25 \%$ have two and $25 \%$ have four, and all have the same buffer space of four slots. We keep the ratio of $N p / N v$ as two and increase the number of peers from 16 to 128.

The adjustment scheme is effective when the system is considerably small. In a large system, pure utilization-based placement works fine, as shown in Figure 12. It confirms that when the system size is considerably large, the adjustment is not necessary. 


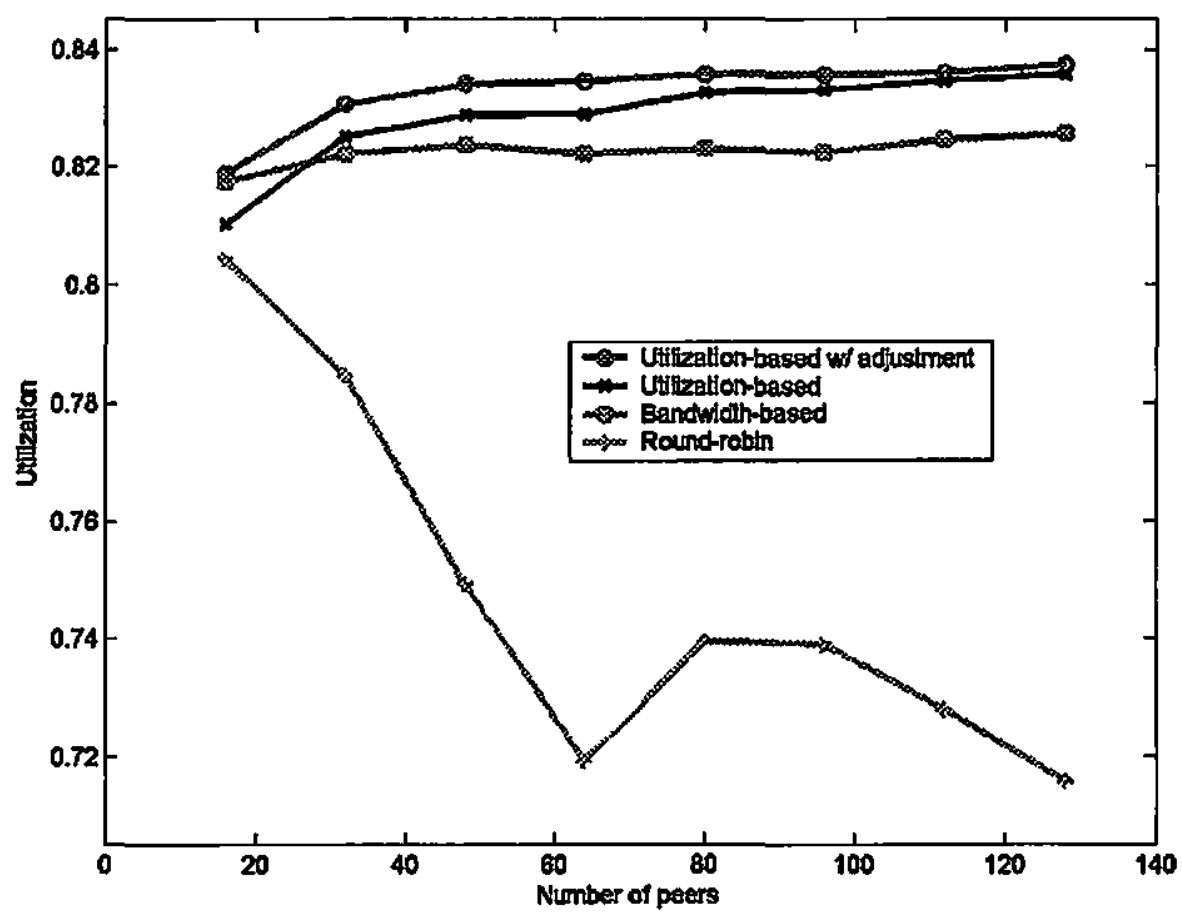

Figure 12: Comparison of utilization-based placement $w /$ adjustment and other placements w/o adjustment

\section{IV.B.3 Effect of System Load}

In this session, we examine the effect of the system load on the performance of the proposed placement schemes. We use a medium size system configuration with 70 videos and 140 supplying peers. Then we choose three combinations of classes of peers, shown in Table 7, to test the performance of the different placement schemes with the load varying from 0.2 to 1.8 . All peers in set 1 have the same size of buffer space. Peers in set 2 have same upstream bandwidth. Peers in set 3 have the same ratio of $u / b$. 
Table 7: Setting for Varying Load Simulation

\begin{tabular}{|c|l|l|l|}
\hline & & $u$ & $b$ \\
\hline \multirow{4}{*}{ Set 1 } & Class 1 (50\%) & 1 & 4 \\
\cline { 2 - 4 } & Class 2 (25\%) & 2 & 4 \\
\cline { 2 - 4 } & Class 3 (25\%) & 3 & 4 \\
\hline \multirow{4}{*}{ Set 2 } & Class 1 (50\%) & 2 & 2 \\
\cline { 2 - 4 } & Class 2 (25\%) & 2 & 4 \\
\cline { 2 - 4 } & Class 3 (25\%) & 2 & 8 \\
\hline \multirow{3}{*}{ Set 3 } & Class 1 (50\%) & 2 & 6 \\
\cline { 2 - 4 } & Class 2 (25\%) & 3 & 8 \\
\cline { 2 - 4 } & Class 3 (25\%) & 4 & \\
\hline \multirow{2}{*}{$N v$} & 70 & & \\
\hline \multirow{2}{*}{$N p$} & 140 & from 0.2 to 1.8 with step 0.2 & \\
\hline Load & & & \\
\hline
\end{tabular}

Table 8 shows the maximum and minimum difference of the utilization to round-robin placement and the corresponding load. Both utilization-based and bandwidth-based placements excel round-robin ones the most when classes of peers have different ratio of $u / b$ (i.e. set $1 \& 2$ ). The maximum difference occurs when the system load is one, while the minimum difference occurs when the load is the smallest in the configuration, 0.2 . This is because when the system load is very low, the competition for the upstream bandwidth becomes smaller. Therefore, we suggest applying utilization-based or bandwidth-based placement schemes when the system load is considerably high, for example 0.4 . Figure 13 shows significant difference when the system load is above 0.4 with the configuration of set 1 .

Table 8: Max \& Min Difference of the Utilization to Round-robin in Percentage 


\begin{tabular}{|l|l|r|r|r|r|}
\hline & & Max difference & At load & Min difference & At load \\
\hline \multirow{3}{*}{ Utilization-based } & Set 1 & 8.51 & 1.0 & 0.02 & 0.2 \\
\cline { 2 - 6 } & Set 2 & 11.71 & 1.0 & 0 & 0.2 \\
\cline { 2 - 6 } & Set 3 & 0.71 & 1.0 & 0 & 0.2 \\
\hline \multirow{3}{*}{ Bandwidth-based } & Set 1 & 8.10 & 1.0 & 0.21 & 0.2 \\
\cline { 2 - 6 } & Set 2 & 12.05 & 1.0 & 0.12 & 0.2 \\
\cline { 2 - 6 } & Set 3 & 0.27 & 1.0 & 0 & 0.2 \\
\hline
\end{tabular}

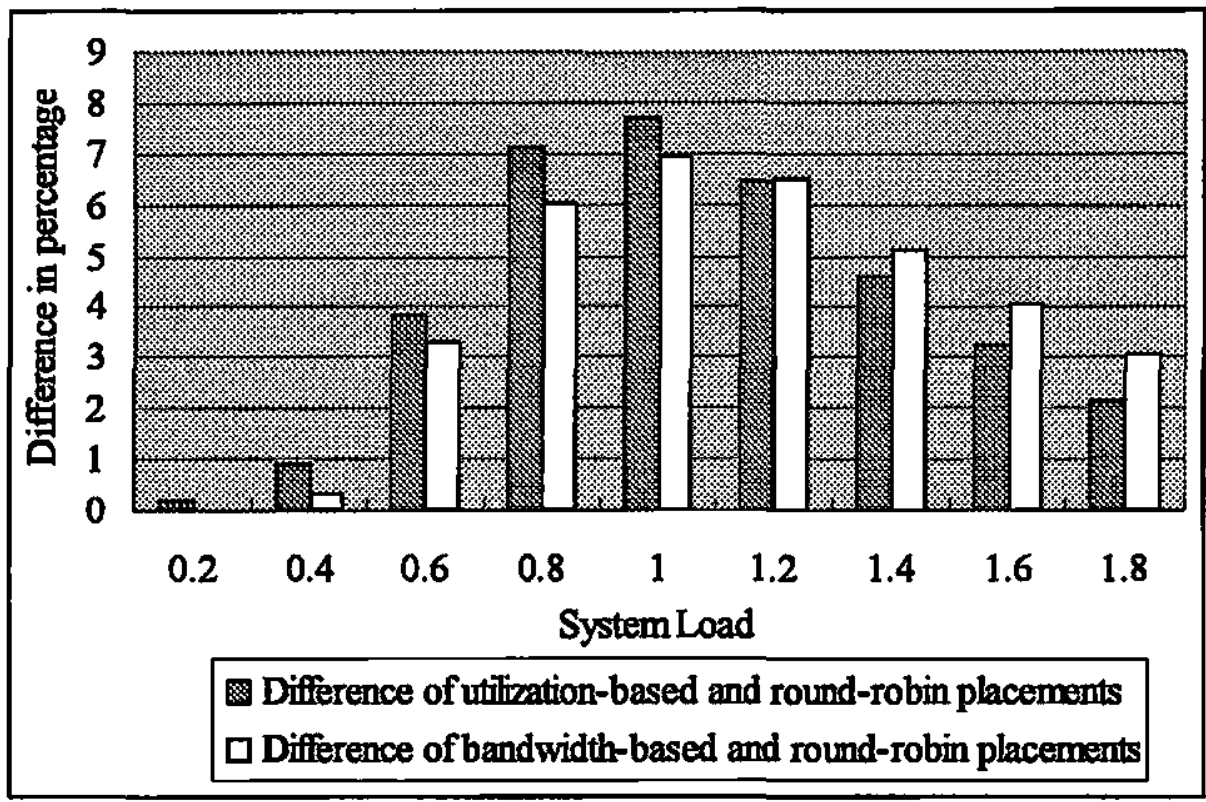

Figure 13: Comparison of utilization to round-robin scheme, set 1

On the other side, when the classes of peers have the same ratio of $u / b$ like set 3 , we do not see much difference. Actually, this is because all of these three placements operate efficiently. When the system is below 0.8 , the rejection rate is close to zero, shown in Figure 14. In addition, the utilization in set 3 is the highest when we compare with the same placement scheme, for example, utilization-based, shown in Figure 15. 


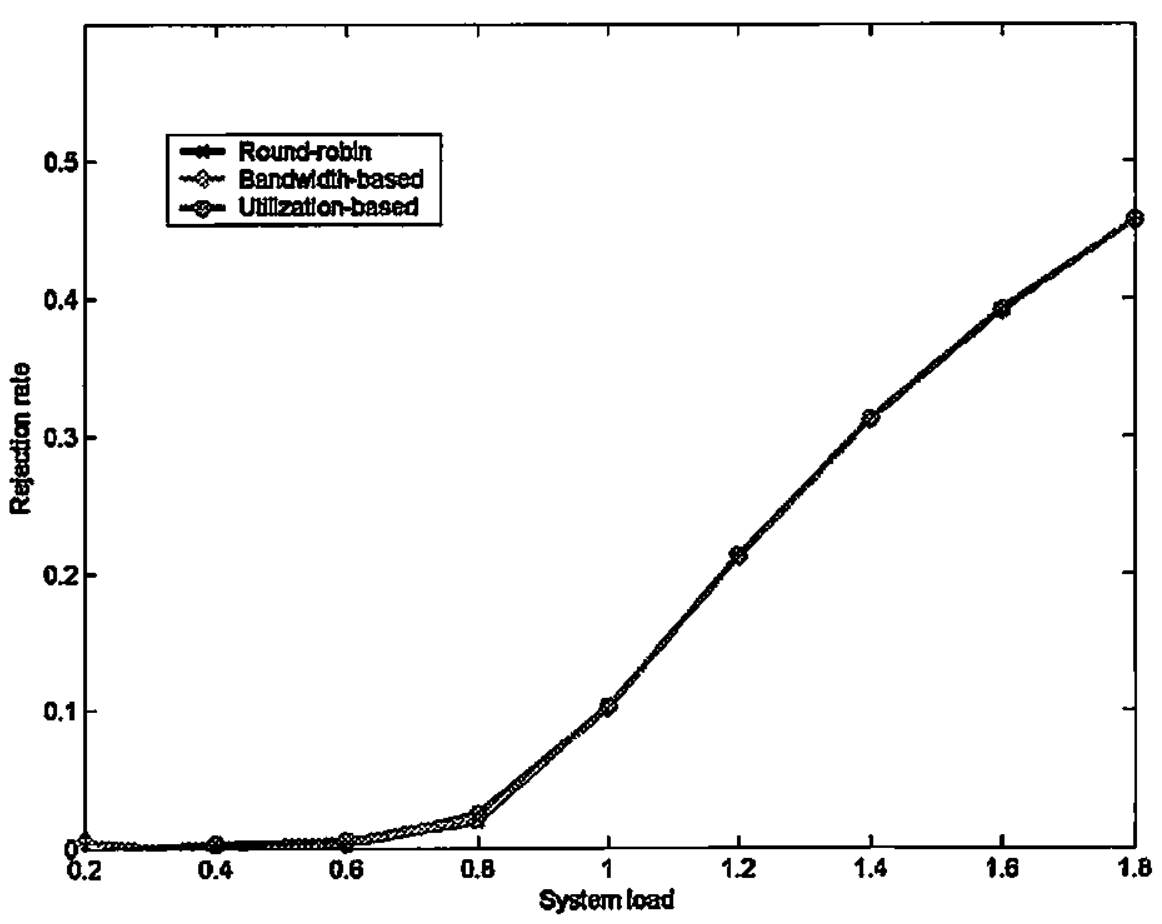

Figure 14: Rejection rate of the three placements for set 3

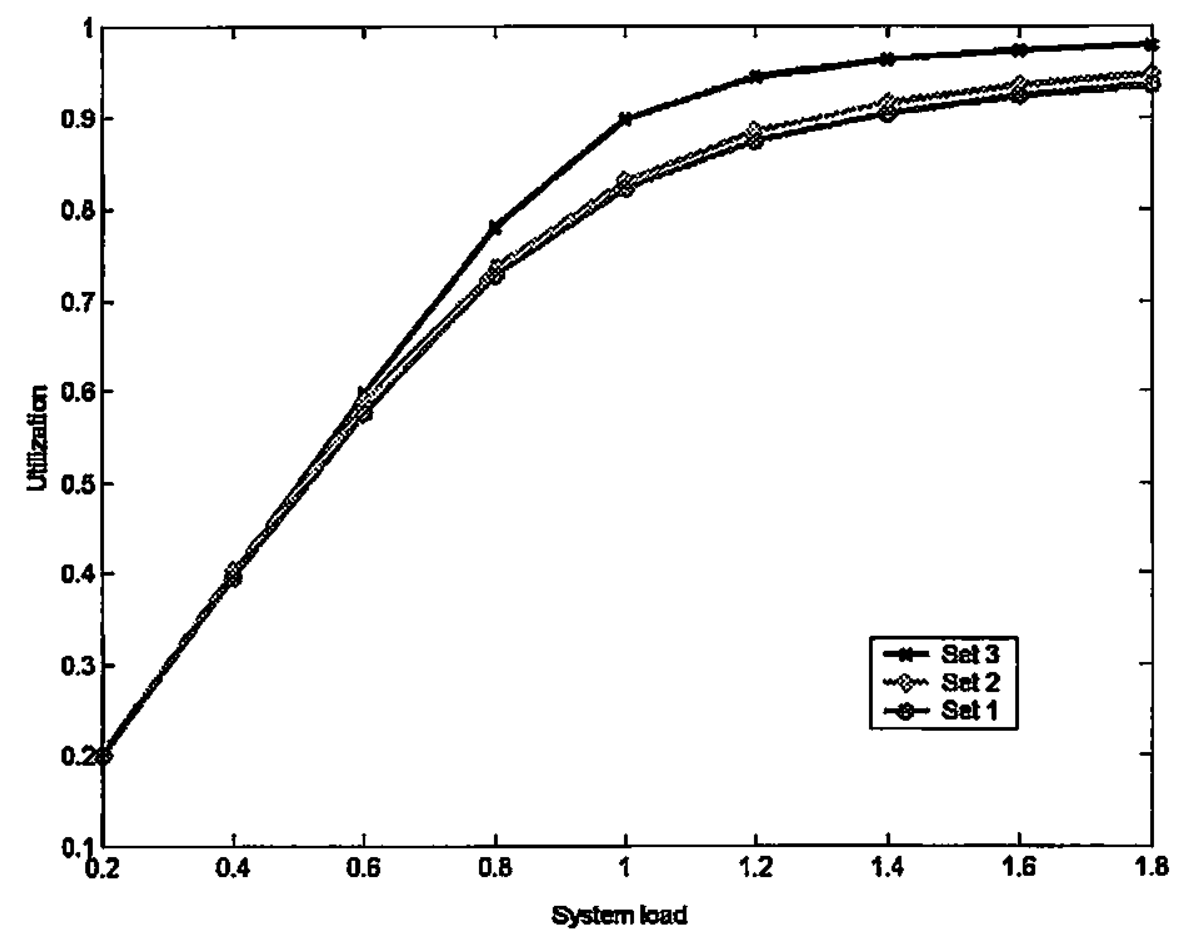

Figure 15: Comparison of utilization-based placements with set 1, 2 \& 3 


\section{IV.B.4 Consideration of the Peer Reliability}

Here we compare the proposed placement schemes with round-robin when considering the reliability of the supplying peers. Same as the previous experiment, we use a medium size system configuration with 70 videos and 140 supplying peers. The supplying peers are classified into two classes: $75 \%$ are DSL peers and $25 \%$ are broadband peers. The DSL peers have smaller upstream bandwidth - 1 upstream channel, while the broadband peers have 4. Assume all peers have 4 buffer slots. DSL peers have a lower reliability, so we vary their reliability from 0.5 to 0.9 with steps of 0.1 and fix the reliability of broadband as 0.95 .

We first see how much improvement can be observed if we use the modified utilization-based instead of original utilization-based scheme. Figure 16 shows a trend that larger differences in the reliability of the two classes achieve more improvement.

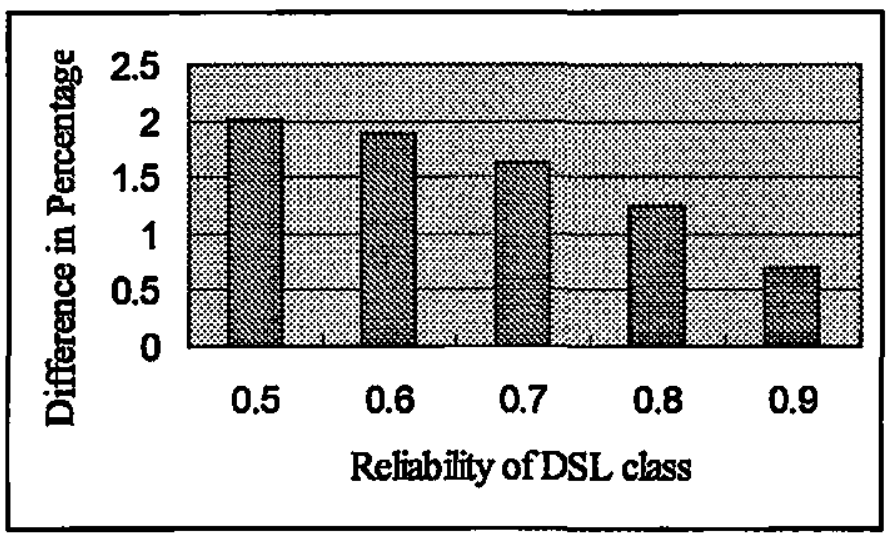

Figure 16: Comparison of modified utilization-based and non-modified

Next, we compare to the round-robin placements. As we expected, the modified utilization-based placements perform much better than the round-robin ones, as shown in Figure 17. 


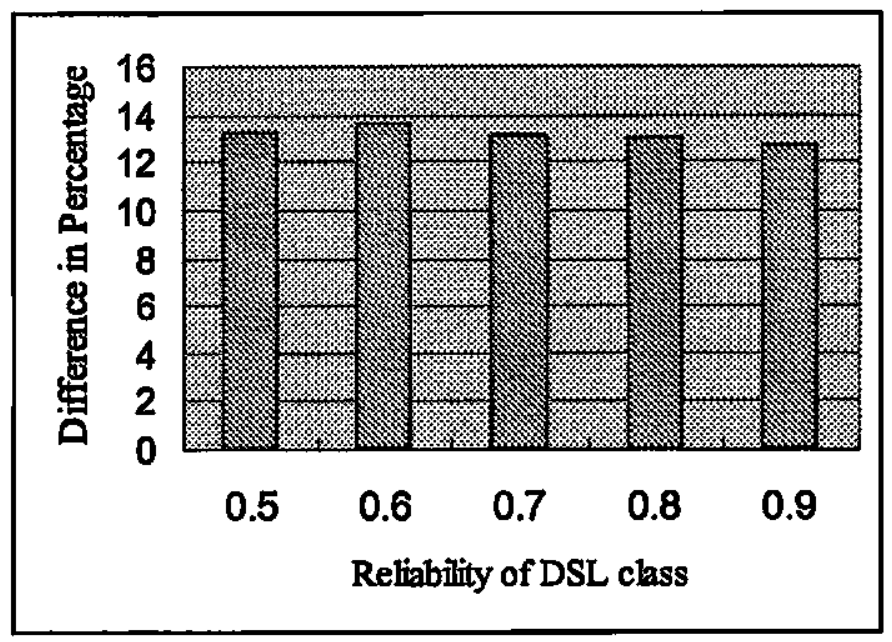

Figure 17: Difference of the utilization of utilization-based and round-robin placements in percentages 


\section{CHAPTER V \\ CONCLUSION}

\section{V.A Discussion}

In this paper, we investigate various placement schemes in order to better utilize peers' upstream bandwidth In Chapter III, we propose two effective placement schemes: bandwidth-based and utilization based. We further improve the proposed placements with the adjustment scheme. Finally, we modify the proposed placement scheme with consideration of the peers' reliability in order to fit into the peers' dynamic nature.

In Chapter IV, we evaluate the proposed placement schemes, bandwidth-based and utilization-based, against round-robin Our analysis and simulation results show the efficacy of the proposed solutions. The advantage is particularly significant when the peers have various but small amounts of upstream channels and buffer size, which is the common case in today's P2P network In this setup, the improvement can be achieved up to $15 \%$. However, there is one configuration where we do not see much improvement: when the ratio of $u / b$ for all the peers is the same. In this specific configuration, all of the three placements perform efficiently, especially when the system load is below 0.8 resulting in a rejection rate is close to zero. The proper number of copies for each video is the key element in this configuration.

When designing the utilization-based placement scheme, we also point out the boundary cases where, at the end, we may not be able to place a copy of a video with the highest remaining bandwidth requirement to the peer with the most available 
upstream bandwidth because this peer already has a copy of that video. To deal with this case, we propose the adjustment scheme - replace a copy or switch copies among peers in order to evenly distribute peers' upstream bandwidth to the videos with respect to their requesting rates. In a small scale distribution system with only 16 peers, the difference of the utilization of the utilization-base with adjustment and without adjustment is $0.9 \%$. When the number of peers increases to 100 or more, the amount of improvement made by the adjustment drops to under $0.2 \%$. Therefore, the adjustment is not necessary for a considerably large system.

We continue to improve our proposed placement schemes by considering the peers reliability. By their nature, peers are not reliable - they may disconnect from the network unpredictably. In addition, different types of peers have different reliability. Broadband peers may be more stable than the DSL peers, because the network is more reliable. If we know the statistic probability regarding the types of peers connected to the network, we should take this into account when designing the placement. For example, the peers' upstream bandwidth and access rate alone is not enough to identify the peer with the most remaining bandwidth. Together with peers connecting probability, we can estimate the peers' remaining bandwidth and the videos' remaining required bandwidth more accurately, so that we can assign the video copies more properly. In a medium size system where the reliability of different types of peers varies, the placement with the consideration of the reliability has a higher utilization than the one without the consideration by $2 \%$, which leads to a total of $14 \%$ improvement over round-robin placement. 
It appears that utilization-based and bandwidth-based have similar performances, (generally speaking, the former performs slightly better than the latter in most cases); however, they have their own advantages in different situations. The complexity of bandwidth-based algorithm is less than utilization-based. The complexity of utilization-based algorithm is $\mathrm{O}\left(N p^{2}\right)$, while the complexity of bandwidth-based is $\mathrm{O}(N p \cdot N v)$. Because, in each iteration of the bandwidth-based scheme, all copies of one video are assigned; while in each iteration of the utilization-based scheme, only one copy is assigned. On the other side, utilization-based is more suitable when the supplying peers are not stable (i.e. they connect and disconnect often). When a new peer joins the collaborative network, the utilization-based scheme can dynamically determine which video copy it should buffer. Unlike utilization-based, bandwidth-based has to figure out the number of copies for each video first. To integrate the advantages of these two schemes, we can initialize the collaborative network with bandwidth-based scheme, and then maintain the network with utilization-based.

\section{V.B Future Work}

Currently, our proposed solutions are not optimal, and we will continue to improve them. This will be beneficial to other similar resource scheduling problems. In addition, our analysis still requires a large amount of resources (i.e. memory space) to a degree that it is unable to handle a large system. We will contimue to simplify our analysis in order to analyze large systems. Hopefully, this will shed some light on 
finding the optimal solution.

We will also design a policy to determine the buffer size of the peers. We have done some research and found out that buffering more video copies does not always benefit the whole system. Therefore, we want to design a policy to determine the minimum required buffer size without degrading the system performance.

Ultimately, we will extend our placement schemes to other peers streaming settings to provide more reliable and quality-controlled services for video-on-demand requests. This may be a great benefit to the content service providers such as Google Video [18] and YouTube [19]. This technique will help to improve the performance to meet the ever-growing demands for video contents through the Internet. 


\title{
Appendix A: Definitions and/or denotations
}

\author{
8 The requesting rate of video $j$ \\ Cs The number of sessions that the CDN server can support simultaneously \\ $C_{p} \quad$ The number of sessions that the supplying peer network can support \\ simultaneously \\ $N_{V} \quad$ The total number of videos \\ $N_{p} \quad$ The total number of supplying peers \\ $b_{i} \quad$ The number of buffer slots of supplying peer $i$ \\ $u_{i} \quad$ The maximum number of upstream channels of supplying peer $i$ \\ $N_{j} \quad$ The maximum possible number of upstream chamnels available for video $j$ \\ $\eta \quad$ The access rate per upstream channel of video $j$ \\ $a_{i} \quad$ The sum of access rate per upstream channel of all video copies stored in \\ peer $i$ \\ t) The target required number of upstream channels for video $j$. \\ wj The number of upstream channels that video $j$ has obtained. \\ sj The remaining required number of upstream channels for video $j$. \\ $\rho_{l} \quad$ The utilization of the supplying peer $i$ \\ $P \quad$ The overall.utilization \\ C Vector, number of copies for video $1 \ldots N v$ \\ $c_{i, t} \quad$ The video of the copy that placed at the $t$ th buffer slot of supplying peer $i$ \\ $\Psi \quad$ Matrix of $c_{i, t}$ \\ $\eta(i, t, j)$ The indicator of whether $c_{i, t}$, the copy that placed at the $t$ th buffer slot of \\ supplying peer $i$ is a copy of video $j$
}




\section{Appendix B:}

\section{Pseudocode for the Proposed Placement Schemes}

\section{BANDWIDTH-BASED $(N p, U, B, \eta$ :}

Imput: $N p$ : the number of the peers

$N v$ : the number of the videos

$U:$ array of the upstream bandwidth for the peers

$B$ : array of the buffer size for the peers

7. array of the arrival rate for the videos

Y. matrix of the placement of videos copies at peers

Parameter:

$G[g]$ : set of peers belong to a class indexed by $g$

$C: \quad$ array of the number of copies for the videos

1. D initiate $\Psi$, set to 0 if the cell is indicated as a buffer slot, $0 / w-1$

2. $\Psi \leftarrow-1$

3. for $i$ from 1 to $N p$

4. for $n$ from 1 to $B[i]$

5. $\Psi[i, n] \leftarrow 0$

6. $\triangleright$ group the peers with the same number of upstream units and buffer slots to one class

7. $\left.I \leftarrow \operatorname{sort}\left(B,{ }^{\prime} \mathrm{a}^{\prime}, U, \mathrm{a}^{\prime}\right)\right)^{*}$

8. $g \leftarrow 1$

9. $G[g] \leftarrow \varnothing$

10. for $i$ from 2 to $N p$

11. If $U[I[i]]=U[I[i-1]]$ and $B[I[i]]=B[I[i-1]]$

12. $G[g] \leftarrow G[g] \chi i$

13. else

- $I=\operatorname{sort}\left(X,{ }^{\prime} a^{\prime} \mid\right.$ 'd', $Y$, ' $a$ '|'d', ...), sorts the element of $X$ in 'a' (ascending) or ' $d$ ' (descending) order, if there are elements of $X$ with same values, sorts them by the corresponding value in $Y$ in ' $a$ ' (ascending) or ' $d$ ' (descending) order, and so on, finally return the array of the indices 
14.

$g \leftarrow g+1$

15.

$$
G[g] \leftarrow \varnothing
$$

16. $\triangleright$ process each class

17. g_max $\leftarrow g$

18. for $g$ from 1 to $g \_\max$

19. $\triangleright$ Compute $C$

20. $i \leftarrow$ any element in $G[g]$

21. factor $\leftarrow \operatorname{size}(G[g]) * B[i] / \operatorname{sum}(\lambda)$

22. for $j$ from 1 to $N v$

23.

$$
\left.C[j] \leftarrow \operatorname{round}(\pi j]^{*} \text { factor }\right)
$$

24. $S \leftarrow f_{\mathfrak{S}}(\Psi, U, B, 7)$

25. $\quad\left[s \_\max , j\right] \leftarrow \max (S)^{*}$

26. $\quad S$ Done $\leftarrow \varnothing$

27. $\triangleright$ Assign copies of video $j$ to the sorted peers

28. while $\left|S \_D o n e\right|<N y$

29. $\triangleright$ sort the peers by $a$

30. $\quad A \leftarrow f_{0}(\Psi, U, B, \lambda$

31. $I \leftarrow \operatorname{sort}\left(A,{ }^{\prime} \mathrm{a}\right)$

32. for $n$ from 1 to $C[j]$

33. $\quad i \leftarrow I n]$

34.

$$
p o s \leftarrow \operatorname{search}(\Psi[i,:]=0)^{\dagger}
$$

35.

$$
\Psi[i, p o s] \leftarrow j
$$

36.

$S \_$Done $\leftarrow S \_$Done $x j$

37. $S \leftarrow f_{5}(\Psi, U, B, 7)$

38. $\quad J \leftarrow \operatorname{sor}(S$, 'd')

39. for $n$ from 1 to $N v$

40.

$$
j \leftarrow J[n]
$$

41.

$$
\text { if } j \notin S \_ \text {Done }
$$

$[y, i]=\max (X)$ returns the index $i$ of the maximum value $y$ in $X$

$t i=\operatorname{search}(X=k)$ returns the index of the entry with value $k$ in the vector $X$. 
42. $\quad\left[s \_\max , j\right] \leftarrow \max (S)$

43. return $\Psi$

UTILIZATION-BASED $(N p, U, B, 7)$ :

Input: $\quad N p$ : the number of the peers

$N v$. the number of the videos

$U$ : array of the upstream bandwidth for the peers

$B$ : array of the buffer size for the peers

7. array of the arrival rate for the videos

$\Psi$. matrix of the placement of videos copies at peers

Parameter:

$G[g]$ : set of peers belong to a class indexed by $g$

C: array of the number of copies for the videos

$V:$ array of the number of copies having buffered for the peers

1. $\triangleright$ initiate $\Psi$, set to 0 if the cell is indicated as a buffer slot, o/w -1

2. $\Psi \leftarrow-1$

3. for $i$ from 1 to $N p$

4. for $n$ from 1 to $B[i]$

5. $\Psi[i, n] \leftarrow 0$

6. $\triangleright$ initiate $V$

7. $V \leftarrow B$

8. $\triangleright$ iteration

9. total_buffer $\leftarrow \operatorname{sum}(B)$

10. for $t$ from 1 to total_buffer

11. $\triangleright$ select video $j$ with the highest remaining bandwidth

12. $S \leftarrow f_{s}(\Psi, U, B, 7)$

13. $J \leftarrow \operatorname{sort}(S$, 'd')

14. for $n$ from 1 to $N v$

15. $j \leftarrow J n]$

16. $\triangleright$ select peer $i$ with the most remaining bandwidth 

17.
$A \leftarrow f_{a}(\Psi, U, B, \lambda$
18.
$I \leftarrow \operatorname{sort}(V$, 'd', $U$, 'd', $A$, ' 'a')
19.
for $i$ from 1 to $N p$
20.

$$
\text { If } 5 \operatorname{find}(\Psi[i,:]=j)^{\circ}
$$
21.

$$
\operatorname{pos} \leftarrow \operatorname{search}(\Psi[i,:]=0)
$$
22.
$\Psi[i, p o s] \leftarrow j$
23.
$\eta[i] \leftarrow V[i]+1$
24.
break

\section{5. return $\Psi$}

ADJUSTMENT( $\Psi, U, B, \lambda$ :

1. $d \leftarrow f_{D}(\Psi, U, B, \lambda)$

2. do

3. $\Psi_{R} \leftarrow$ REPLACEMENT $(\Psi, U, B, \eta$

4. $d_{R} \leftarrow f_{D}(\Psi, U, B, \lambda)$

5. $\Psi_{E} \leftarrow \operatorname{EXCHANGE}(\Psi, U, B, \lambda$

6. $d_{E} \leftarrow f_{D}(\Psi, U, B, \lambda)$

7. $\quad$ if $\min \left(d_{R}, d_{E}\right)<d$

8. $\quad d \leftarrow \min \left(d_{R}, d_{E}\right)$

9. if $\min \left(d_{R}, d_{E}\right)=d_{R}$

10. $\Psi \leftarrow \Psi_{R}$

11. else

12. $\Psi \leftarrow \Psi_{E}$

13. else

14. break

15. loop

16. return $\Psi$

\footnotetext{
" find $(X=k)$ retums true if find the value $k$ in $X$, false otherwise
} 
REPLACEMENT $(\Psi, U, B, \lambda$

1. $S \leftarrow f_{B}(\Psi, U, B, \lambda$

2. $\left[s_{-} \max , J\right] \leftarrow \max (S)$

3. $\left[s_{-} \min , k\right] \leftarrow \min (S)^{*}$

4. $A \leftarrow f_{a}(\Psi, U, B, \lambda$

5. weight $\leftarrow$ ceiling $(\max (A))+1$

6. $\triangleright$ find the peer $i$ with a copy of video $k$ but $j$ and with the fewest buffer slots and lowest total access rate per upstream channel if there are multiple pees with the fewest buffer slots

7. for $i$ from 1 to $N p$

8. If find $(\Psi[i,:]=k)$ and $5 \operatorname{find}(\Psi[i,:]=j)$

9. score $[i] \leftarrow B[i] \times$ weight $+A[i]$

10. else

11. score $[i] \leftarrow \mathbf{I N F}$

12. [score_min, $i] \leftarrow \min ($ score $)$

13. $\triangleright$ if the peer is found, replace the copy of video $k$ with $j$.

14. If score $[i] \neq \mathbb{N}$ F

15. $\quad p o s \leftarrow \operatorname{search}(\Psi[i, j]=k)$

16. $\Psi[i, p o s] \leftarrow j$

17. retura $\Psi$

$\operatorname{EXChANGE}(\Psi, U, B, \lambda$

1. $S \leftarrow f_{s}(\Psi, U, B, \lambda$

2. $\left[s_{-} \max , j\right] \leftarrow \max (S)$

3. $\left[s \_\min , k\right] \leftarrow \min (S)$

4. $A \leftarrow f_{a}(\Psi, U, B, \lambda)$

5. $\triangleright$ find the peer $i k$ with a copy of video $k$ but $j$ and with the fewest upstream chamnels and highest total access rate per upstream channel if there are multiple

$\cdot[y, i]=\min (X)$ returns the index $i$ of the minimum value $y$ in $X$ 
pees with the fewest upstream channels

6. weight $\leftarrow$ ceiling $(\max (A))+1$

7. for $i$ from 1 to $N p$

8. If find $(\Psi[i,:]=k)$ and $\operatorname{Sfind}(\Psi[i,:]=j)$

9. $\quad$ score $k[i] \leftarrow U[i] \times$ weight $-A[i]$

10. else

11. score_k[i] $\leftarrow \mathbf{N F}$

12. [score_min, $i k] \leftarrow \min \left(s c o r e \_k\right)$

13. $\triangleright$ find the peer $i j$ with a copy of video $j$ but $k$ and with the most upstream channels and lowest total access rate per upstream channel if there are multiple pees with the most upstream channels

14. for $i$ from 1 to $N p$

15. If find $(\Psi[i,:]=j)$ and $\neg$ find $(\Psi[i,:]=k)$

16. score $j[i] \leftarrow U[i] \times$ weight $-A[i]$

17. else

18. score $j[i] \leftarrow-\mathbb{N F}$

19. [score_min, $i]$ ] $\leftarrow \max ($ score_j)

20. If score_k[ik] $\neq \mathrm{INF}$ and score_j$[i j] \neq-\mathrm{INF}$

21. $\quad p o s k \leftarrow \operatorname{search}(\Psi[i k,:]=k)$

22. $p o s j \leftarrow \operatorname{search}(\Psi[i j,:]=j)$

23. $\Psi[i k, p o s k] \leftarrow j$

24. $\Psi[i j, p o s y] \leftarrow k$

25. return $\Psi$ 


\section{Appendix C: Experiment Results}
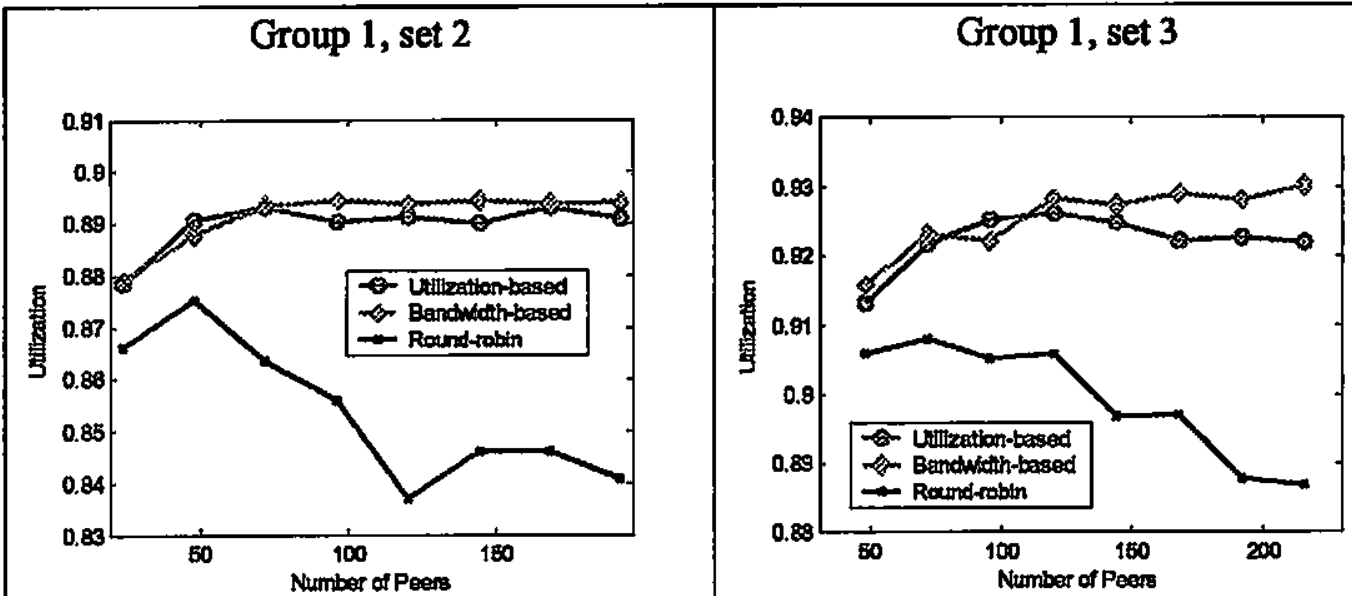

(a) Configuration 1

(a) Configuration 1
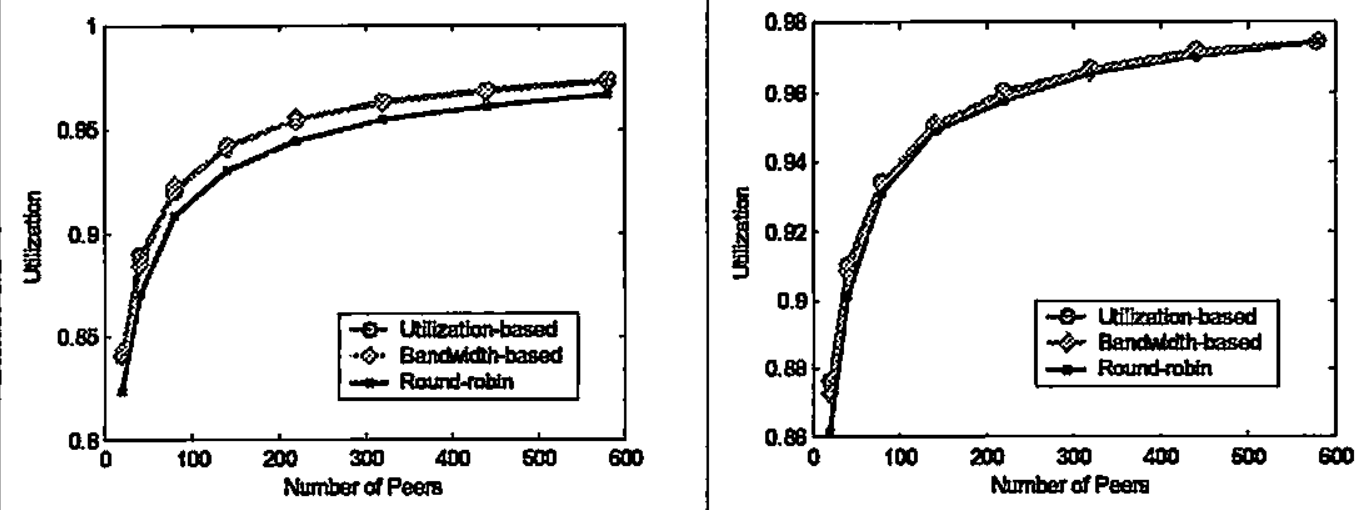

(b) Configuration 2
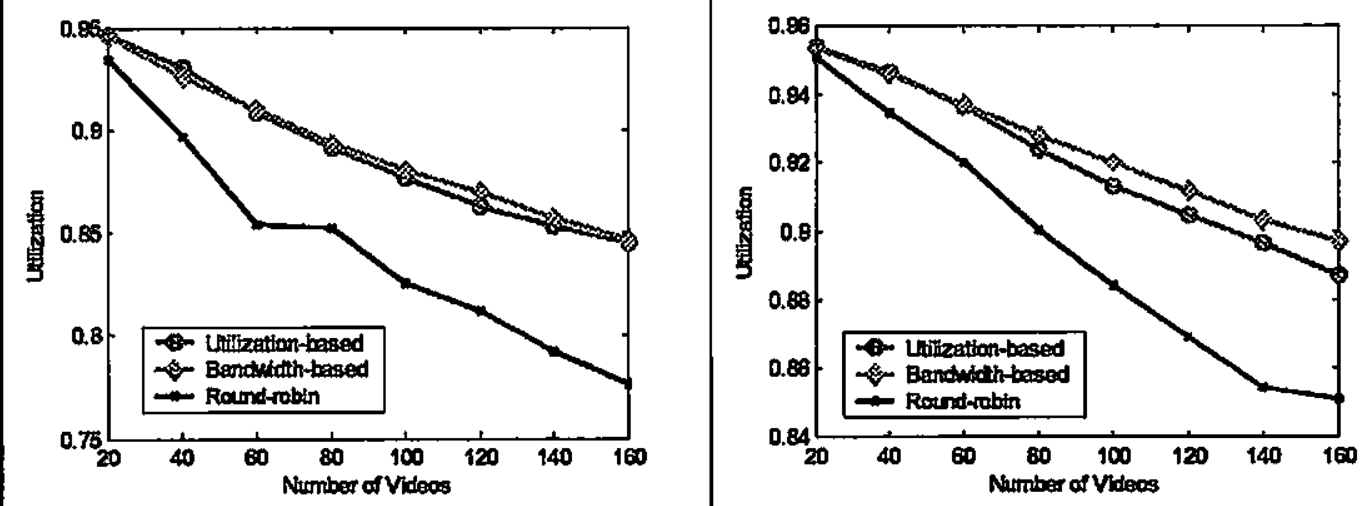

(c) Configuration 3

(c) Configuration 3 


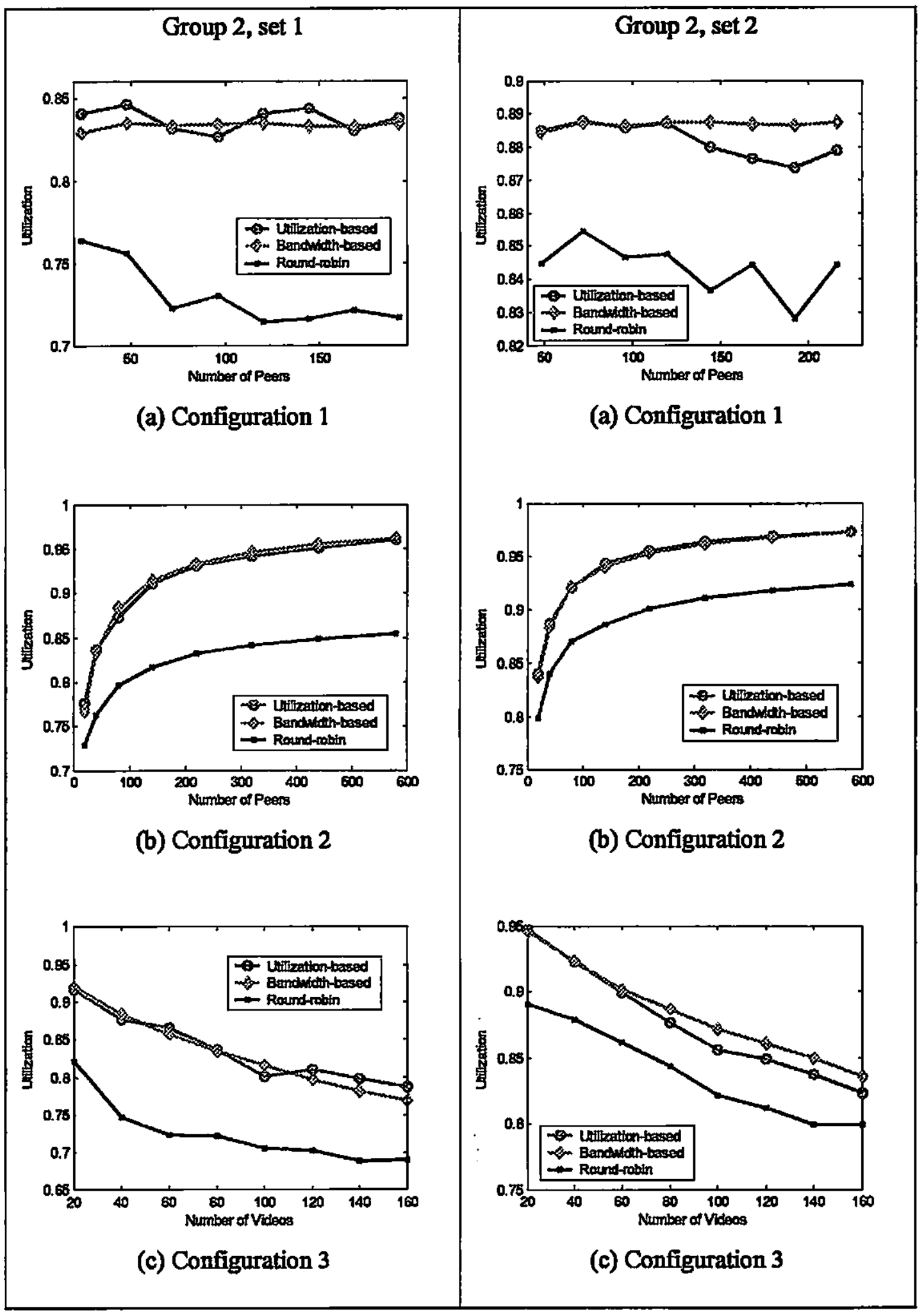




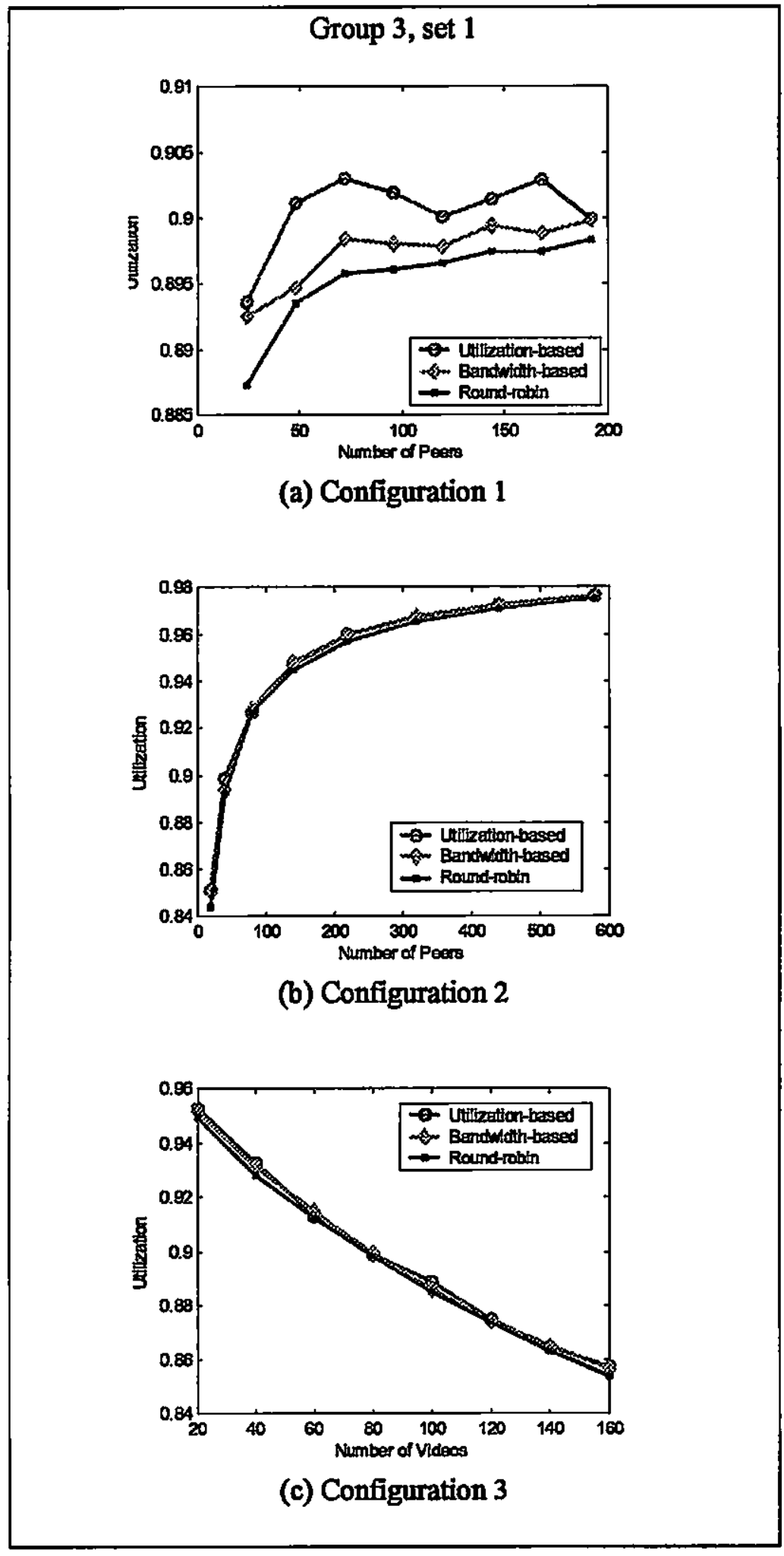




\section{REFERENCES}

[1] A. Albanese, J. Blomer, J. Edmonds, M. Luby, and M. Sudan, "Priority Encoding Transmission" in IEEE trans. Information Theory, Vol.42, pp.1737 - 1744, Nov. 1996.

[2] S. Banerjee, S. Lee, R. Braud, S. Bhattacharjee, and A. Srinivasan, "Scalable Resilient Media Streaming" in Proc. Of NOSSDAV'04,2004.

[3] V. K. Goyal. , "Multiple Description Coding: compression Meets the Network" in IEEE Signal Proc. Mag., vol. 18, pp. 74-93, May. 2001.

[4] P. Chou, H. Wang, and V. Padmanabhan., "Layered Multiple Description Coding" in Packet Video Workshop, Nantes, France, April. 2003.

[5] Y. Dong, E. Kusmierek, D. Duan, and D. Du, "A HYBRID CLIENT-ASSISTED STREAMING ARCHITECTURE: MODELING AND ANALYSIS," in Proc. of IMSA, Aug. 2004.

[6] M. Hefeeda, A. Habib, B. Botev, D. Xu, and B. Bhargava, "Promise: Peer-to-Peer Media Streaming Using CollectCast" in ACM Multimedia 03, Pages 45-54, Nov. 2003.

[7] V. Padmanabhan, H. Wang, P. Chou, and K. Sripanidkulchai, "Distributing Streaming Media Content Using Cooperative Networking" in Proc. Of NOSSDAV '02, Miami, Florida, 2002.

[8] D. Tran, K. Hua, and T. Do, "Scalable Application Layer Multicast" in Proc. Of IEEE INFOCOM, Mar. 2003.

[9] Y. Shen, Z. Liu, S. Panwar, K.W. Ross, and Y. Wang, "Peer-Driven Video Streaming: Multiple Descriptions versus Layering," http://cis.poly.edu/ ross/papers/p2pvideo_July05.pdf.

[10] D. Xu, S. Kulkarni, C. Rosenberg, and H. Chai, "A CDN-P2P hybrid Architecture for Cost-effective Streaming Media Distribution" in Computer Network, Vol.44, Issue.3, pp.353-382, 2004. 
[11]T. Nguyen, A. Zakhor, "Path Diversity With Forward Error Correction (PDF) System For Packet Switched Network", Infocom 2003, IEEE, 2003

[12]S. M. Lui, S. H. Kwok, "Interoperability of Peer-to-Peer File Sharing Protocols", ACM SIGecom Exchanges, Pages 25-33, Vol. 3, Issue 3, 2002

[13]C. H. Ding, S. Nutanong, R. Buyya, "Peer-to-Peer Networks for Content Sharing", Technical Report, GRIDS-TR-2003-7, Grid Computing and Distributed Systems Laboratory, University of Melbourne, Australia, December 2003

[14]J. E. Berkes, "Decentralized Peer-to-Peer Network Architecture: Gnutella and Freenet", University of Manitoba, Winnipeg, Manitoba, Canada, April 2003

[15] "Peer-to-Peer (P2P) and How Kazaa Works", http://www.kazaa,com/us/help/glossary/p2p.htm

[1] K. Tutschku, "A Measurement-Based Traffic Profile of the eDonkey Filesharing Service", Passive and Active Network Measurement, 5th International Workshop, PAM 2004, Antibes Juan-les-Pins, France Apiil 19-20, 2004. Proceedings, LNCS, Vol. 3015/2004.

[17] B. Cohen, "Incentives Build Robustness in BitTorrent", May 2003

[18] http://video.google.com

[19] http://www.youtube.com

[20] S. McCanne, V. Jacobsen, and M. Vetterli, "Receiver-driven layered multicast", ACM SIGCOMM, Ang. 1996.

[21] S. Wenger, "Video Redundancy Coding in H.263+", Workshop on Audio-Visual Services for Packet Networks, September 1997.

[22] V. Vaishampayan and S. John, "Interframe balanced-multiple descriptionvideo compression", IEEE Inter Conf. on Image Processing, Oct. 1999.

[23] A. Reibman, H. Jafarkhani, Y. Wang, M. Orchard, and R. Puri,"Multiple description coding for video using motion compensated prediction", IEEE Inter. Conf. Image Processing, October 1999. 
[24] J. Apostolopoulos, "Error-resilient video compression via multiple state streams", Proc. International Workshop on Very Low Bitrate Video Coding (VLBV'99), October 1999.

[25] J. Apostolopoulos, "Reliable Video Communication over Lossy Packet Networks using Multiple State Encoding and Path Diversity," Visual Communications and Image Processing, January 2001.

[26] J. Apostolopoulos, T. Wong, W. Tan, and S. Wee, "On Multiple Description Streaming with Content Delivery Networks," IEEE INFOCOM, July 2002.

[27] A. Sinha, K. Mitchell, D. Medhi, "Flow-Level Upstream Traffic Behavior in Broadband Access Networks: DSL versus Broadband Fixed Wireless", 2003 IEEE

[28] S. Lee, R. Sherwood, B. Bhattacharjee, "Cooperative Peer Groups in NICE", IEEE, 2003.

[29] YH Chu, J. Chuang, and H. Zhang, "A Case for Taxation in Peer-to-Peer Streaming Broadcast", ACM SIGCOMM'04 Workshop 